Open Access to Pharmaceutical and Medical Research

(C) 2011-18, publisher and licensee JDDT, This is an Open Access article which permits unrestricted non-commercial use, provided the original work is properly cited

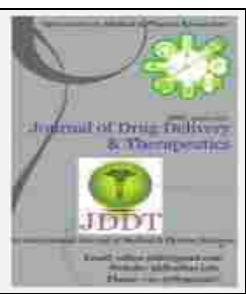

Open $\odot$ Access

Research Article

\title{
GC-MS Analysis of Bioactive Compounds Present in Marketed Herbal Formulation Pykure Capsule
}

\author{
Dr. Seema Firdouse ${ }^{*}$, Humaira Fatima Begum², Dr. Parwez Alam ${ }^{3}$ \\ ${ }^{1}$ Associate Professor, Department of Pharmaceutical Analysis and Quality Assurance, Anwarul Uloom College of Pharmacy, Hyderabad- 500001, \\ Telangana, India. \\ 2 Department of Pharmaceutical Analysis, Anwarul Uloom College of pharmacy, Hyderabad- 500001, Telangana, India \\ ${ }^{3}$ Associate Professor, Department of Pharmacognosy, Shadan College of Pharmacy, Peerancheeru, Hyderabad-500091, Telangana, India
}

\begin{abstract}
It is more acceptable to believe that natural remedies are safer than synthetic subjects that with fewer side effects. The global market demand was increasing due to fusion of herbs. Standardization parameters and evaluation of herbal formulation is essential in order to assess the quality of drug of therapeutic value. This study was carried out in order to determine the bioactive compounds present in the marketed herbal formulation pykure capsule by using the GC-MS instrument. The extract was prepared by using 20 capsules by removing the capsule shell with ethanol solvent by simple maceration method. Capsule extract was analyzed by using Agilent 6890 GC with 59739N MSD and GC -MS equipped with Elite-I fused with silica capillary column (Cpsil $8 \mathrm{cb}: 30 \mathrm{~mm} \times 25 \mathrm{~mm} \times 0.25 \mathrm{~mm}$ ). The result of GC-MS analysis confirmed the presence of 74 compounds.
\end{abstract}

Keywords: Pykure capsule, Ethanolic extract, GC-MS analysis.

Article Info: Received 13 July 2019; $\quad$ Review Completed 17 Aug 2019; $\quad$ Accepted 24 Aug 2019; Available online 15 Oct 2019

Cite this article as:

Firdouse S, Begum HF, Alam P, GC-MS Analysis of Bioactive Compounds Present in Marketed Herbal Formulation Pykure Capsule, Journal of Drug Delivery and Therapeutics. 2019; 9(5-s):73-104 http://dx.doi.org/10.22270/jddt.v9i5-s.3601

*Address for Correspondence:

Dr. Seema Firdouse, Associate Professor, Department of Pharmaceutical Analysis and Quality Assurance, Anwarul Uloom College of Pharmacy, Hyderabad-500001, Telangana, India.

\section{INTRODUCTION}

Herbal medicines can be defined as any medicinal product which contains active ingredients as one or more herbal preparation or herbal substances [1]. Herbal medicine is an ancient form of ailment. The use herbal medicine is increased tremendously over past 2 decades. In this field, there is a significant lack of research data. Since 1990 three volumes of WHO monographs has been published on selected medicinal plants [2]. Herbal medicine has become an important alternative medicine. In today's world it has became popular because of its natural remedies. In many countries, traditional herbal medical system is of economic importance. $80 \%$ of the population of Africa uses herbal drugs in order to meet the health care needs. Asia and America uses herbal medicine because of cultural benefits. In china around $40 \%$ of herbal medicines of all health cares are delivered. About $70-80 \%$ of African and Asian population is using herbal medicine as a primary medicine which is estimated by WHO [3]. Pykure capsules are the pure herbal drugs manufactured by natural Ayurvedic herbs. In the last ten years more than $1,50,000$ patients are treated to the entire patients satisfaction. Each $750 \mathrm{mg}$ of Pykure capsule contains the following ingredients: Terminalia chubularets 0.10 grams, Abutilon indicum - 0.10 grams, Mesua ferrea 0.08 grams, Azadirachta indica - 0.19 grams, Saraca indica 0.12 grams, Cuminum cyminum - 0.05 grams. It is used to treat different types of Ano-rectal disorders [4, 5].

\section{MATERIALS AND METHODS:}

\subsection{Preparation of sample:}

Take 20 capsules, gently remove the cap and body of capsules then transfer all the capsule content in $250 \mathrm{ml}$ volumetric flask. To the above drug contents add $150 \mathrm{ml}$ ethanol, all the contents of the flask should be mixed well with stirrer and tightly closed and should be kept for $24 \mathrm{hrs}$ with continuous shaking. After $24 \mathrm{hrs}$ filter the contents of the flask and discard the solid residue and filtrate is used for analysis. 
2.2. Methods of Analysis of sample by Gas chromatography-Mass spectrometry (GC-MS):

GC-MS analysis of sample was performed by using Agilent 6890 GC with 59739N MSD and GC -MS equipped with Elite I, fused with silica capillary column (cpsil 8cb :30 mm x $25 \mathrm{~mm} \times 0.25 \mathrm{~mm}$ ) composed of $100 \%$ Dimethyl polysiloxane. For GC-MS detection an electron ionization system with ionizing energy of $70 \mathrm{ev}$ was used. Hydrogen $(99.99 \%)$ was used as the carrier gas at a flow rate of $1.5 \mathrm{ml}$ and an injection volume of 2 micro liters. It was employed with split ratio of $10: 1$, Initial injection temp of $40^{\circ} \mathrm{c}$ to final 2800 c. Ion source temp 2300 c. The oven temp was programmed from $1100 \mathrm{c}$ (isothermal for 2 minute) with a rate of $10^{0} \mathrm{c}$ per min to $300^{\circ} \mathrm{c}$. And a scan interval $0.5 \mathrm{sec}$ and fragments from 20-200 Dalton. Total GC running time was 36 minutes. The relative average peak area and retention time, molecular formula with that of molecular weight were obtained. The interpretation on mass spectrum GC-MS was conducted by using the data base of national institute standard and technology (NIST), having 62,000 patterns. The spectrum of unknown component was compared with spectrum of known component stored in NIST library. The name, molecular weight and structure of the components of the sample material were identified [6].

\section{RESULTS AND DISCUSSION:}

\section{GC-MS analysis of ethanolic extract of pykure capsules:}

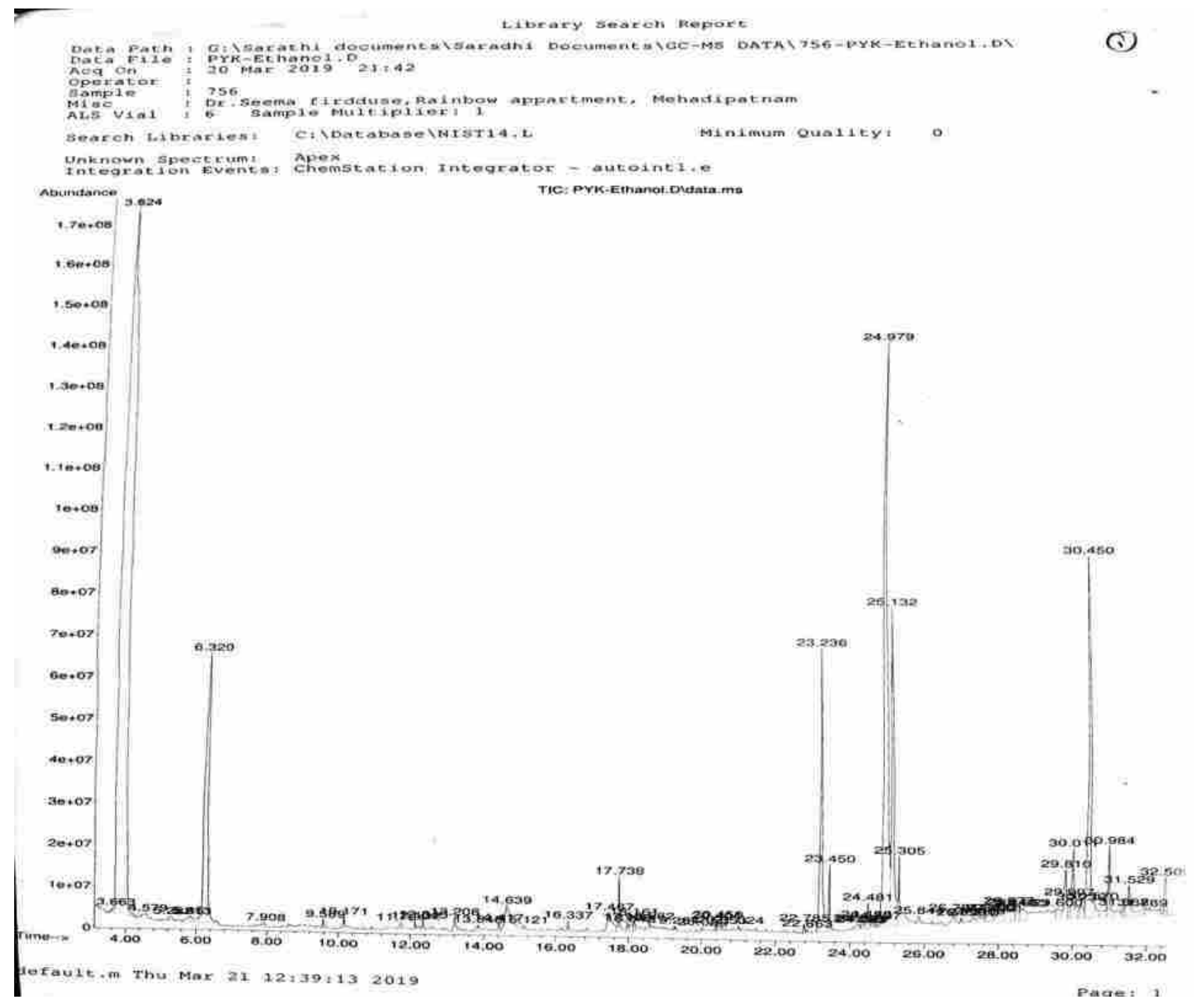

Figure1: GC-MS chromatogram of the ethanolic extract of pykure capsules. 


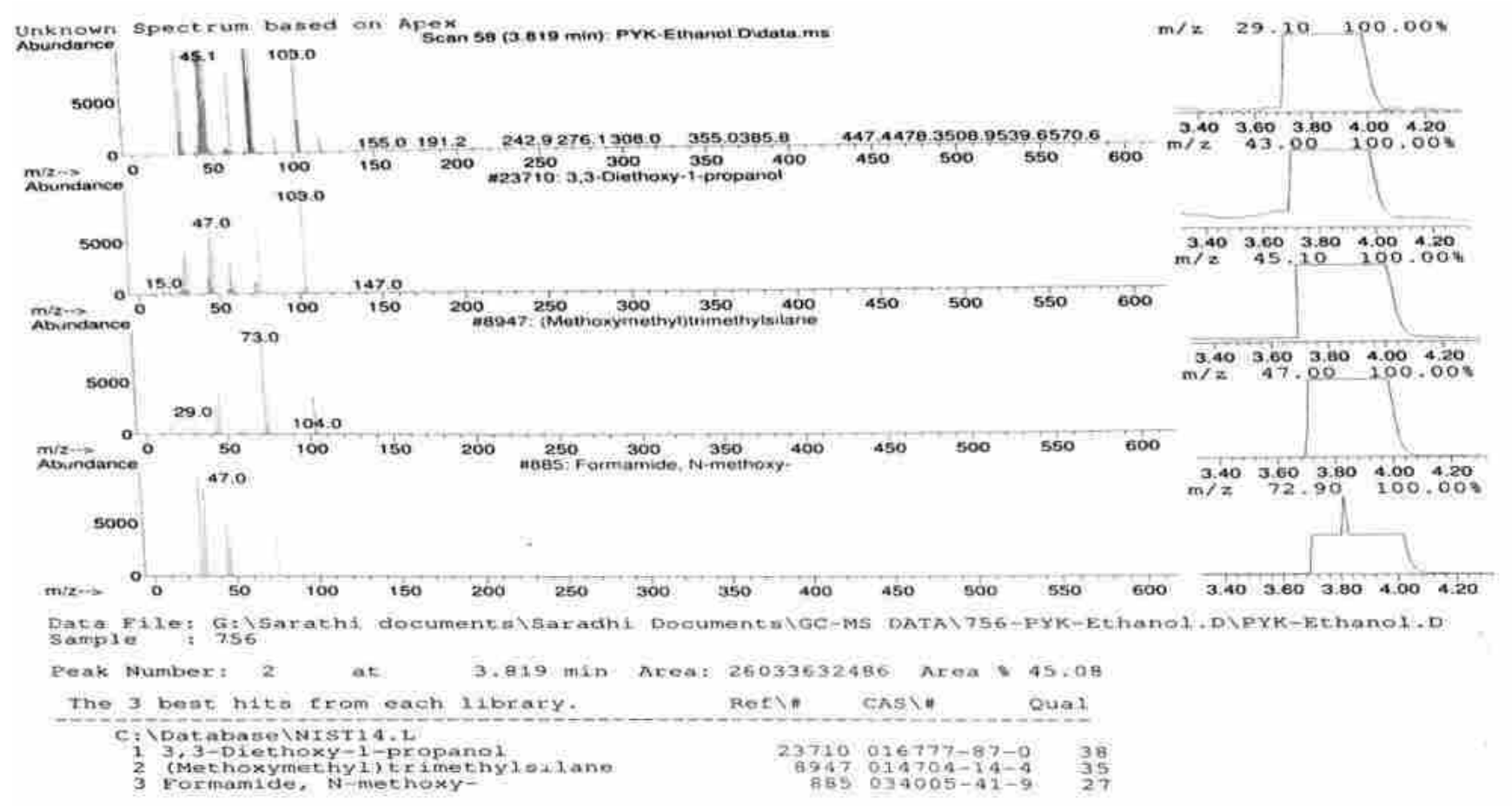<smiles>CCOC(CCO)OCC</smiles><smiles>COC[Si](C)(C)C</smiles>

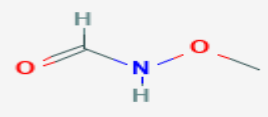

\section{3, 3-Diethoxy-1-propanol (Methoxymethyl) trimethyl silane Formamide, n-methoxy}

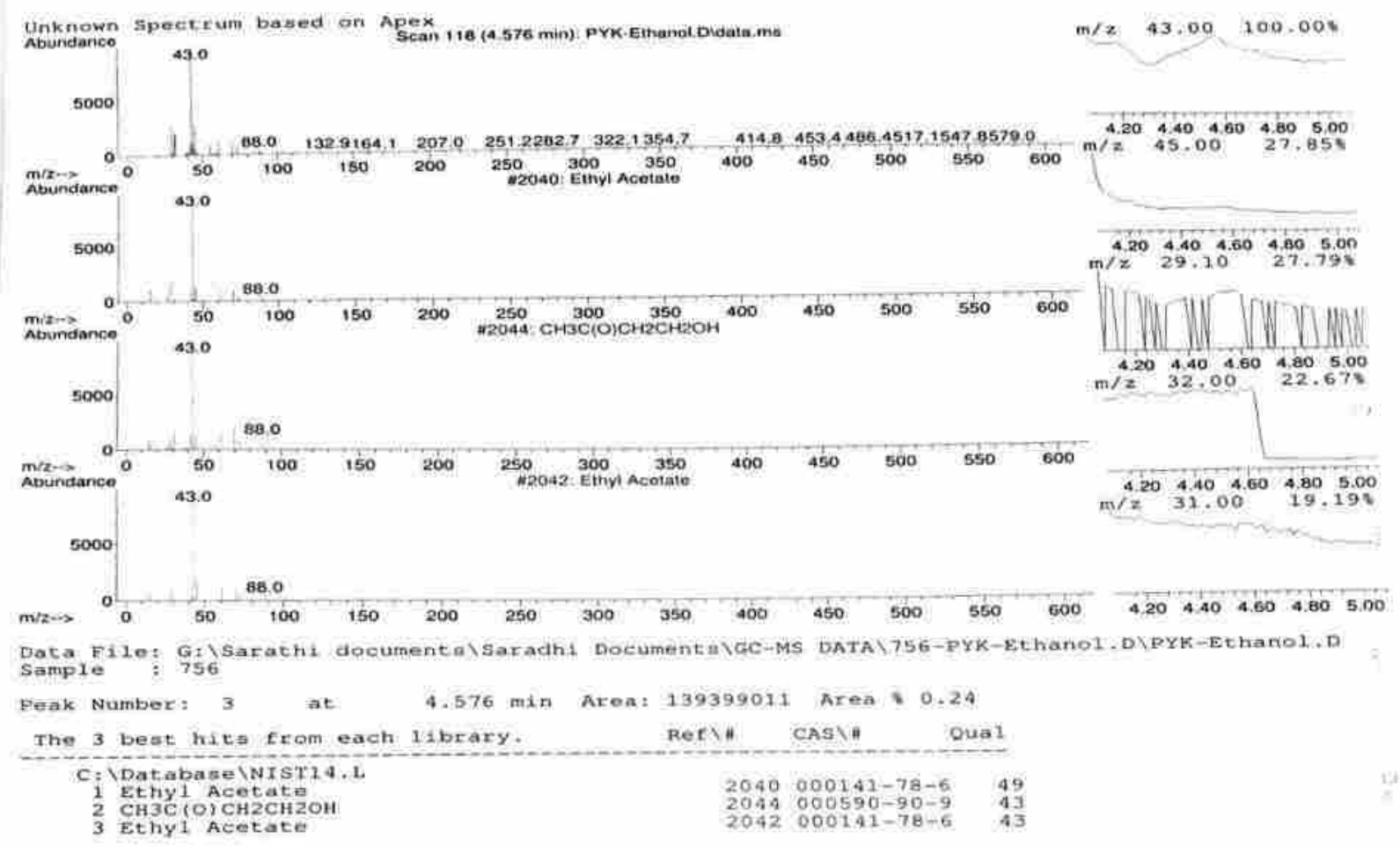<smiles></smiles> 


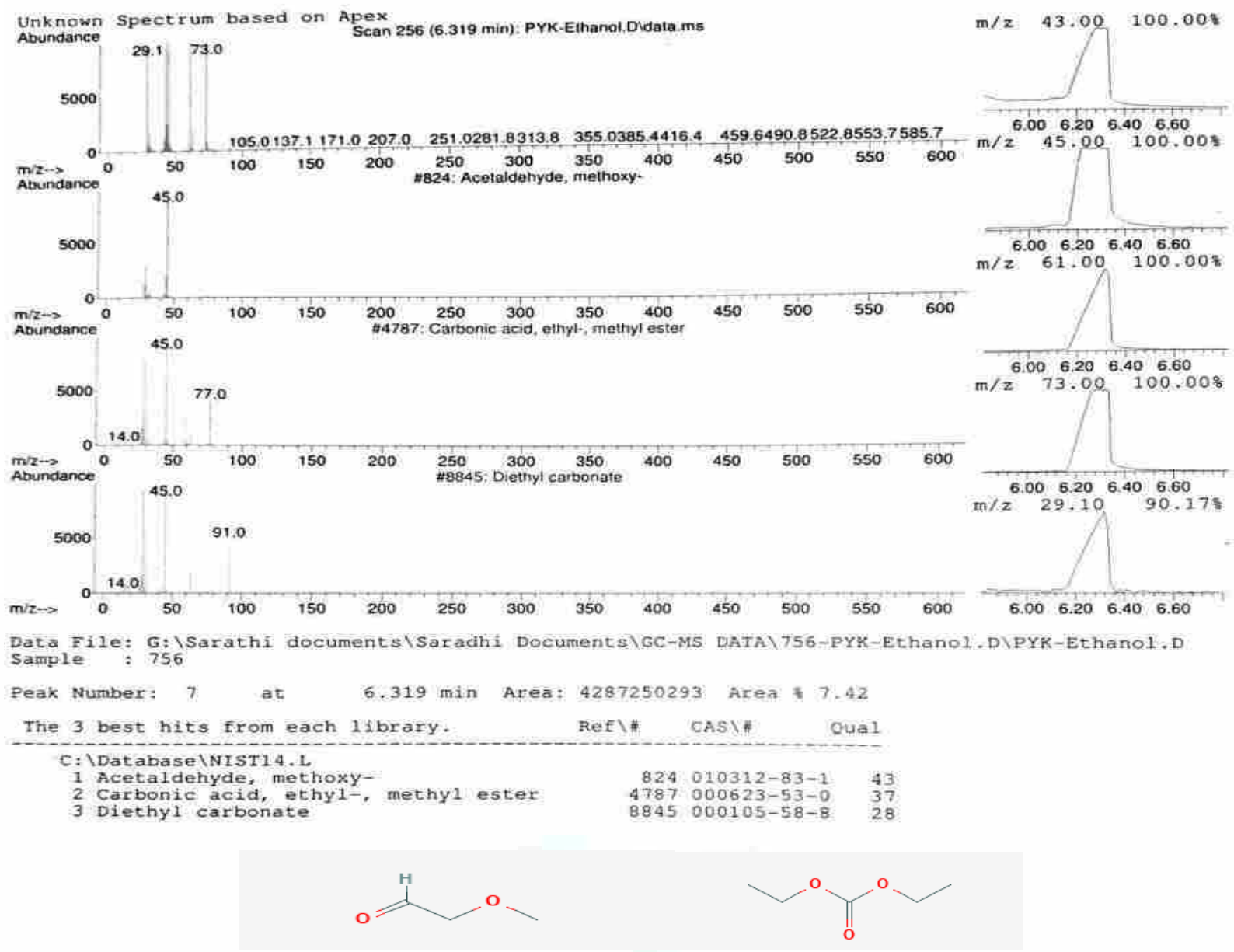

Acetaldehyde, methoxy-

Diethyl carbonate

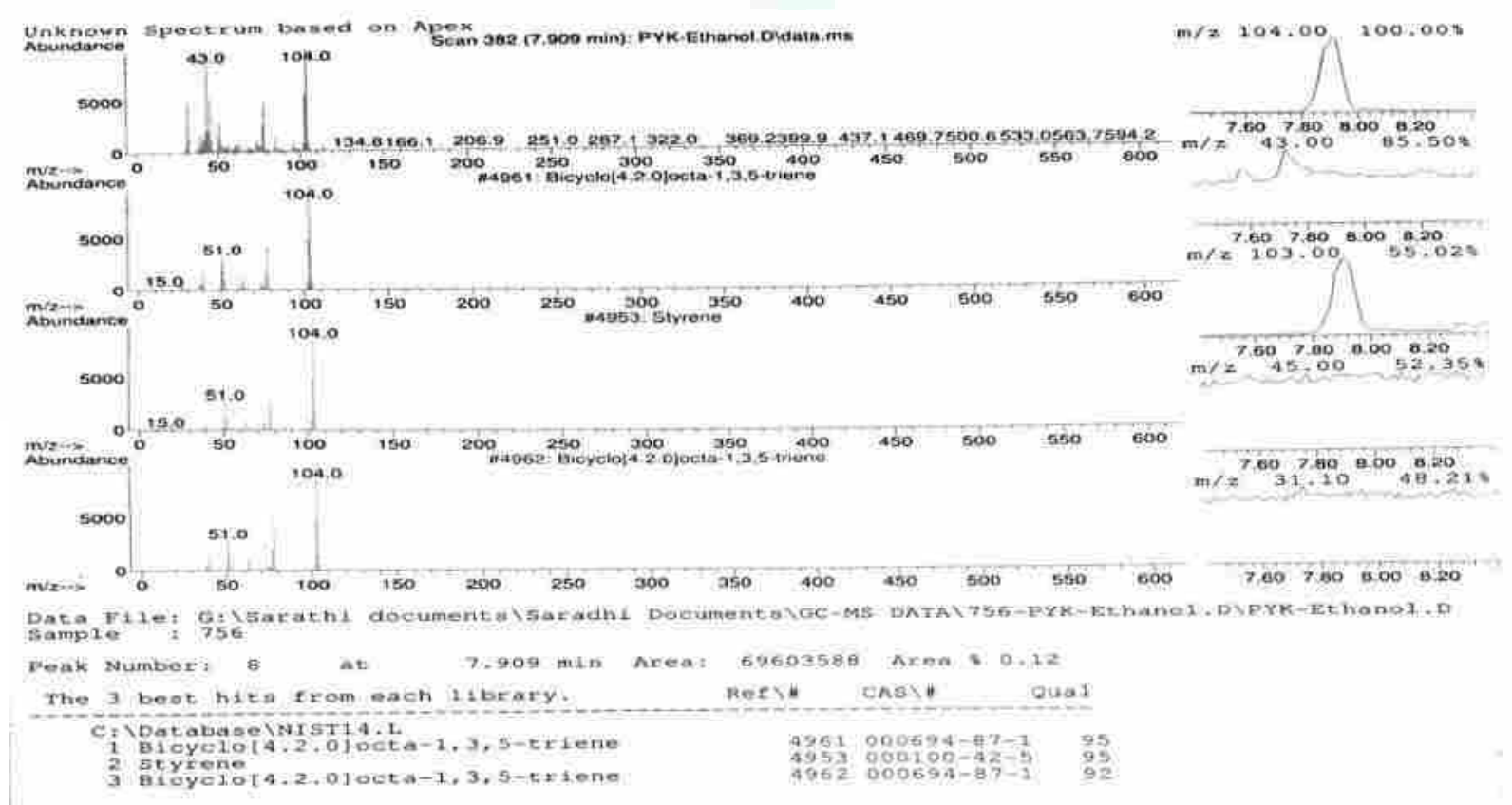

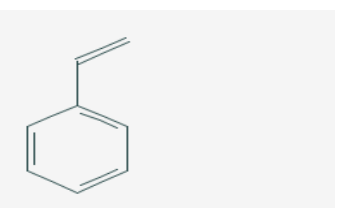

Styrene

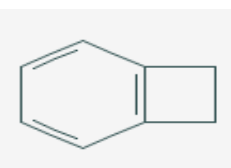

Bicyclo[4.2.0]octa-1,3,5-triene 

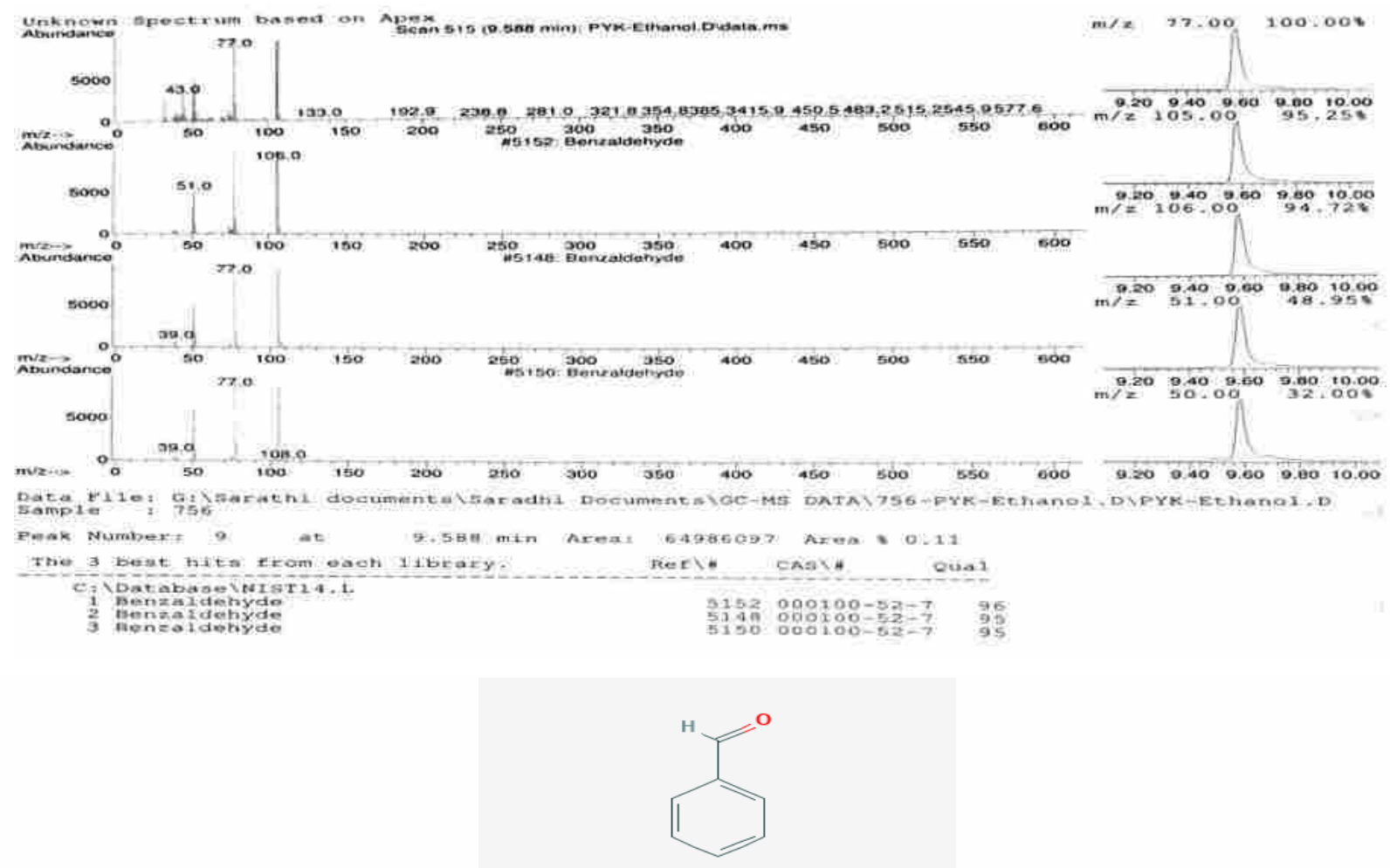

\section{Benzaldehyde}

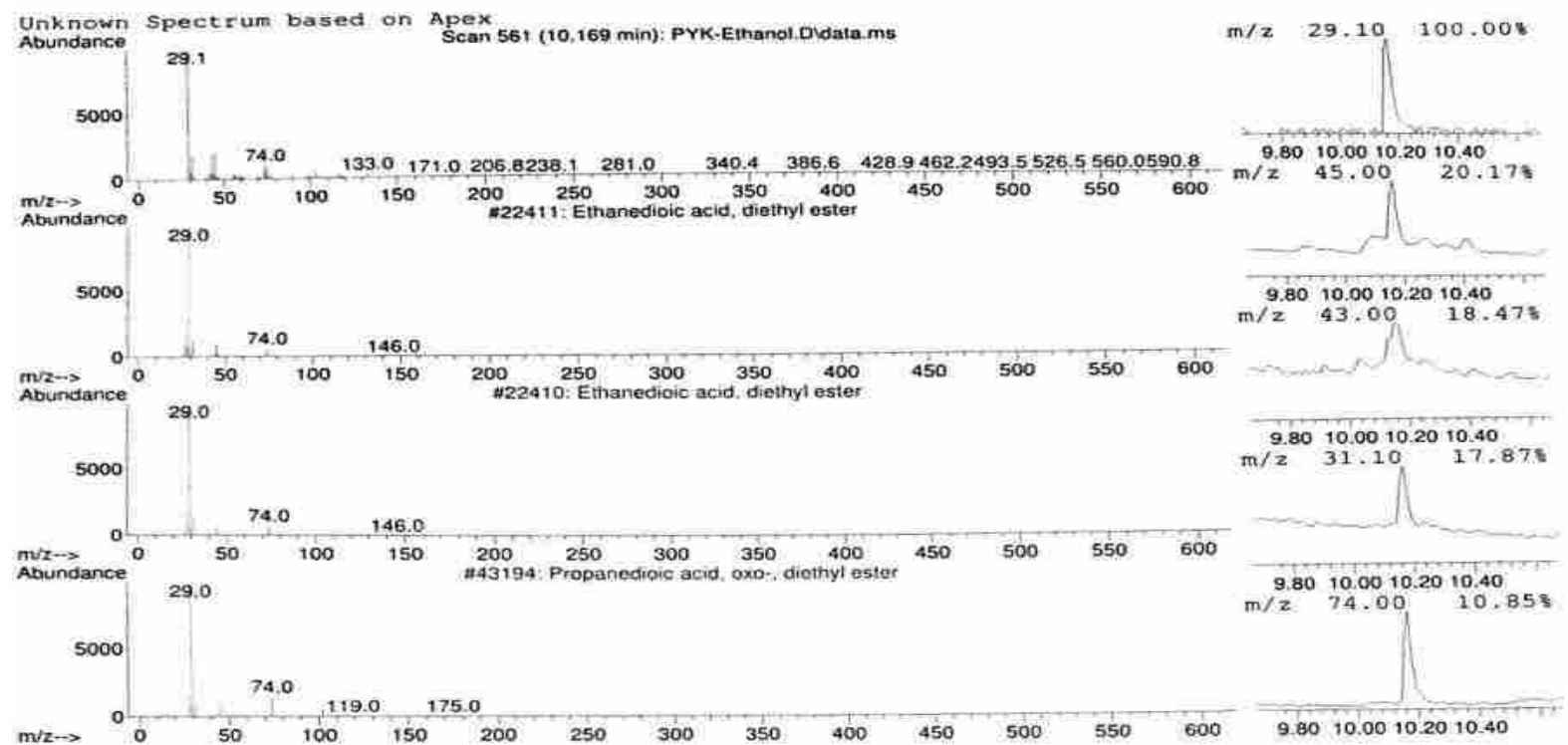

Data File: G: ISarathi documentslSaradhi DocumentslGC-MS DATAI756-PYK-Ethanol.D PYK-Ethanol.D Sample: 756

Peak Number: 10 at $10.169 \mathrm{~min}$ Area: 49955898 Area 0.09

The 3 best hits from each library.

Refl\# CASI\# Qual

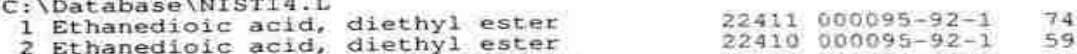

2 Ethanedioic acid, diethyl ester $3 . . .43194000609-09-6 \quad 45$<smiles>CCOC(=O)C(=O)OCC</smiles>

Ethanedioic acid, diethyl ester<smiles>CCOC(=O)C(=O)C(=O)OCC</smiles>

Propanedioic acid, oxo-diethyl ester 


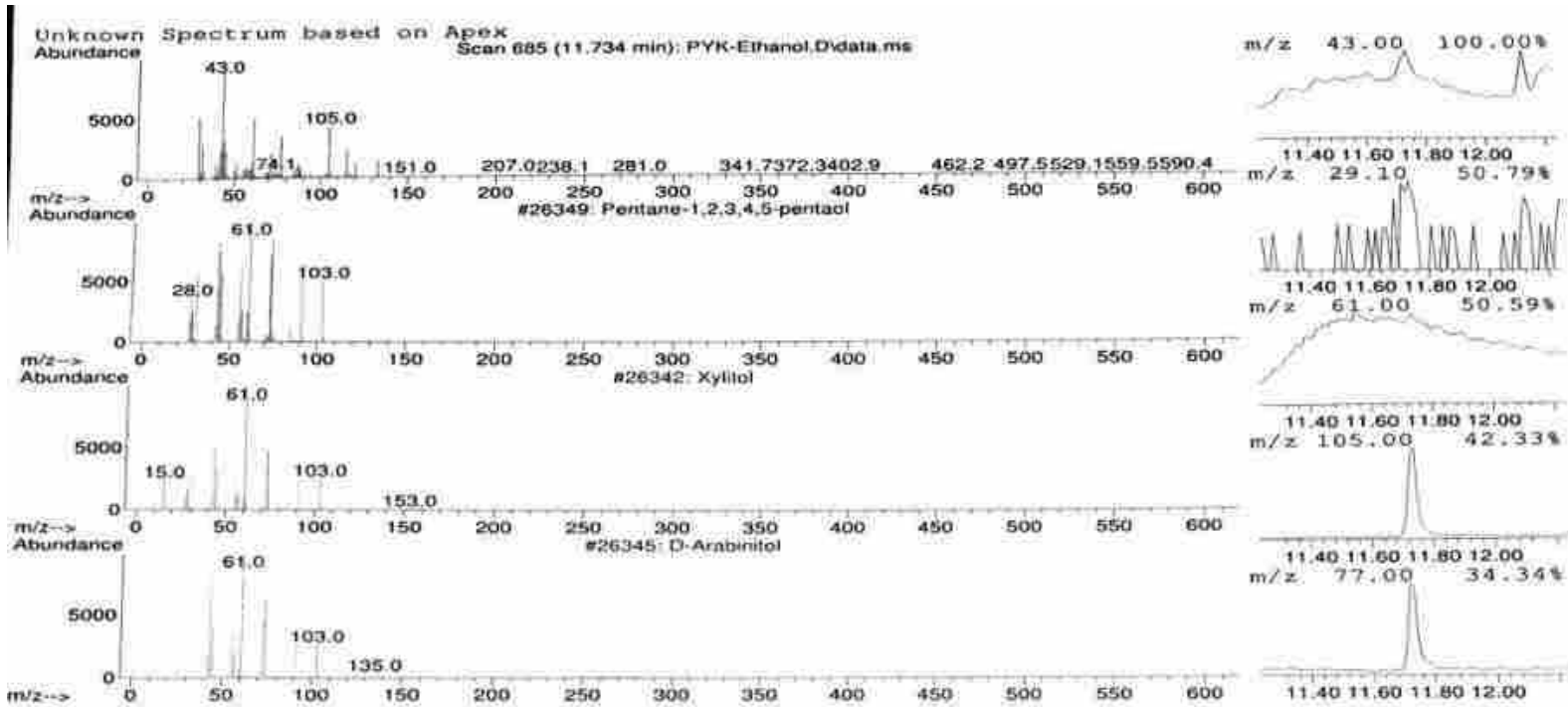

$\begin{array}{llllllllllllll}m / 2 & 50 & 100 & 150 & 200 & 250 & 300 & 350 & 400 & 450 & 500 & 550 & 600 & 11.4011 .6011 .8012 .00\end{array}$

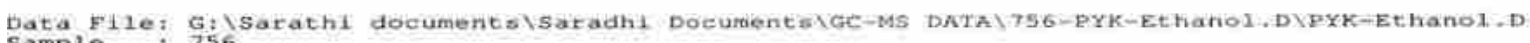
Sample: 756

Peak Number: 11 at $11.734 \mathrm{~min}$ Area; 41861203 Af 10.0 .07

The 3 best hita from each 1 ibrary.

C: Database NNISI14.L

1 Pentane-1, 2, 3, 4, 5-pentao 1

2 Xylitol
3 D-Arabinitol

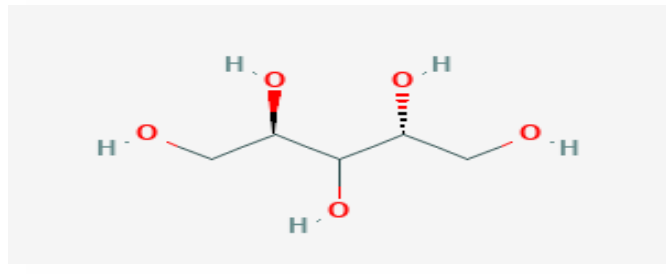

D-Arabinitol

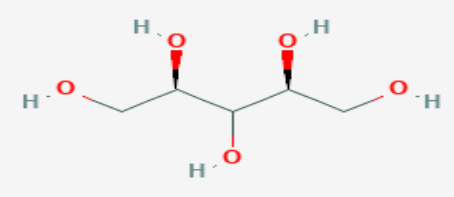

Xylitol

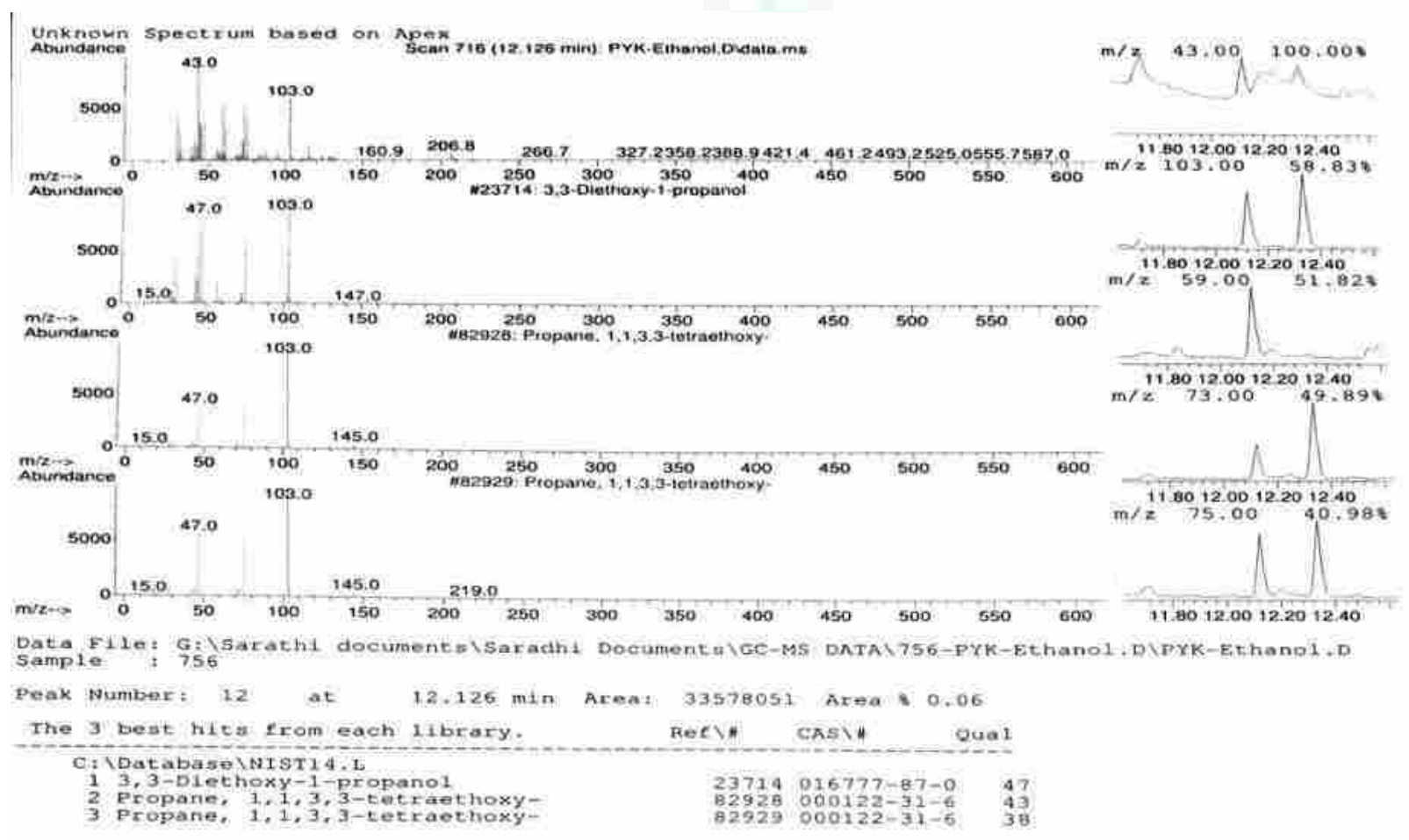

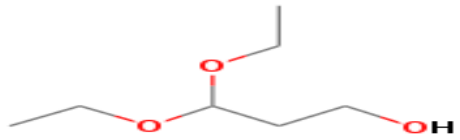

3,3-diethoxy-1-propanol

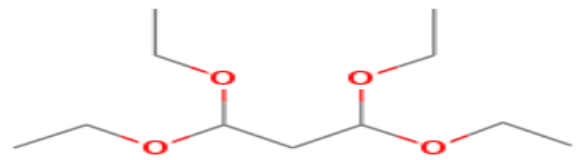

propane, 1,1,3,3-tetraethoxy 


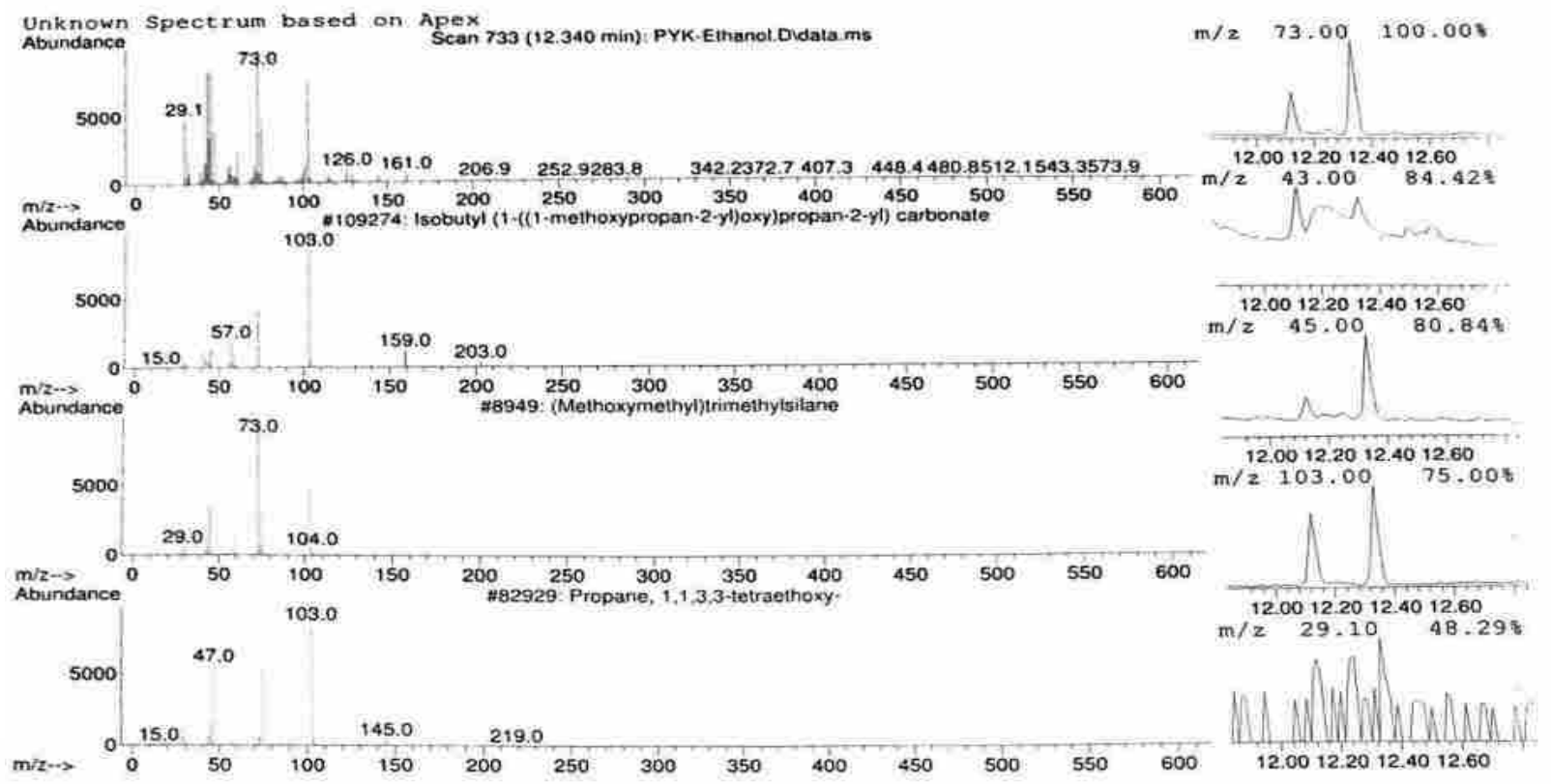
Data File: G: ISarathi documentslSaradhi DocumentslGC-MS DATA 756 -PXK-Ethanol.DVPYK-Ethanol.D
Sample: 756

Peak Number: 13 at $12.340 \mathrm{~min}$ Area: 45758926 Area $\neq 0.08$

The 3 best hits from each library. Refl\# CASl\# Qual

C: IDatabaselNISI 14.1

1 Isobuty $11-(11$-methoxypropan-2-... $\quad 109274 \quad 1000378-28-8 \quad 43$

2 (Methoxymethyl) trimethylsilane $\quad 8949014704-14-4 \quad 40$

3 Propane, 1, 1, 3,3-tetraethoxy- $\quad 82929000122-31-6 \quad 38$

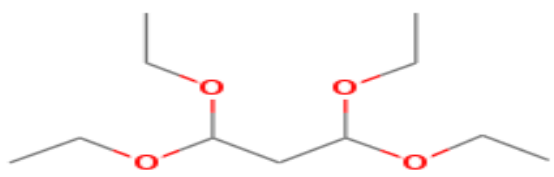

Propane, 1, 1, 3, 3, tetraethoxy 


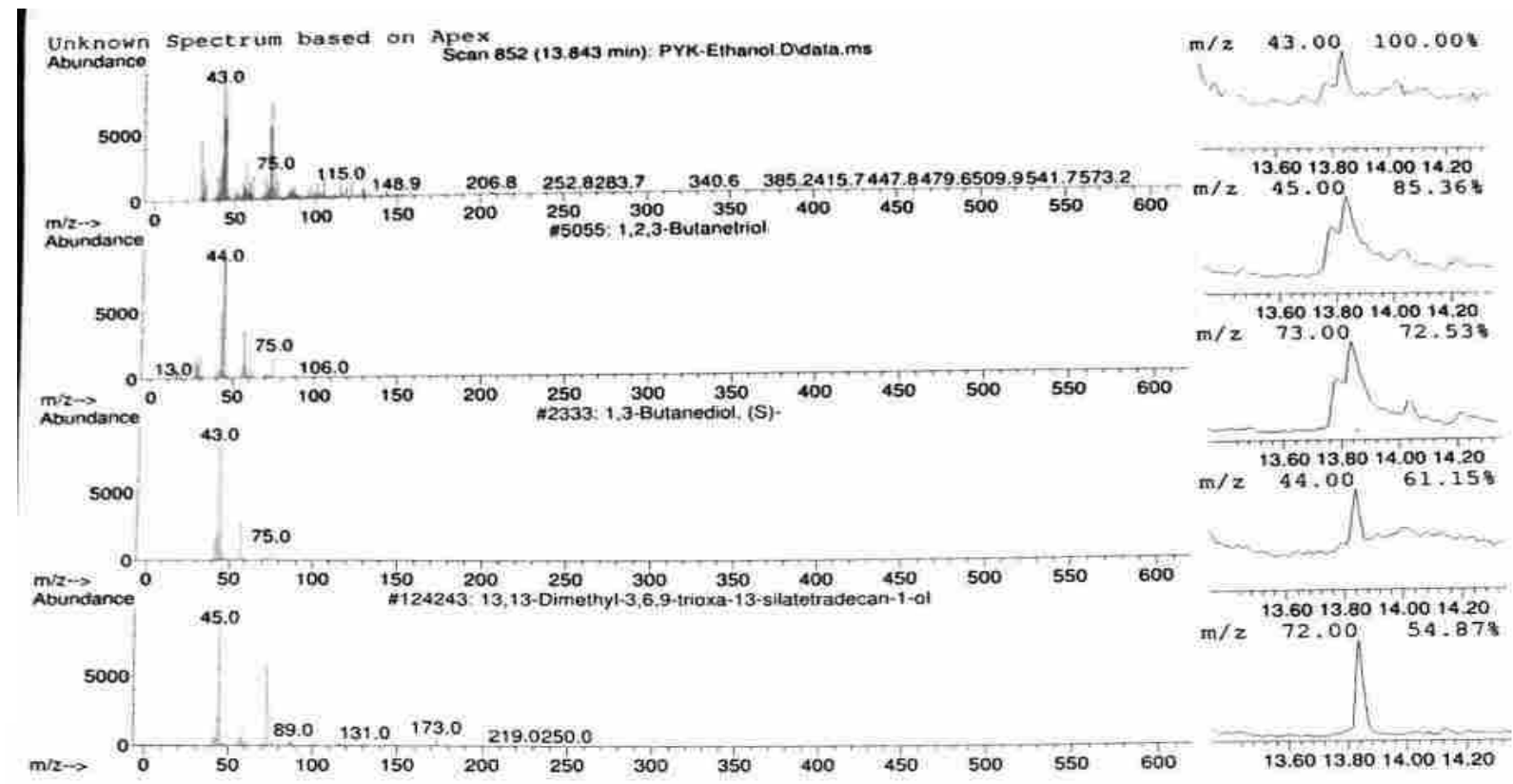

Data File: G: ISarathi documents ISaradhi Documents/GC-MS DATAI756-PYK-Ethanol.DIPYK-Ethanol.D Sample: 756

Peak Number: 15 at $13.843 \mathrm{~min}$ Area: 54096964 Area $\$ 0.09$

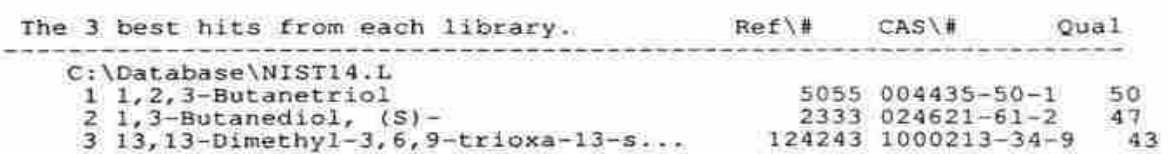

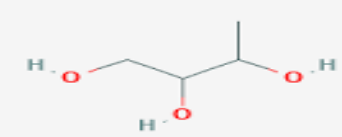

1, 3 butane

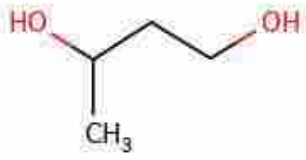

1, 2,3-butanetriol 


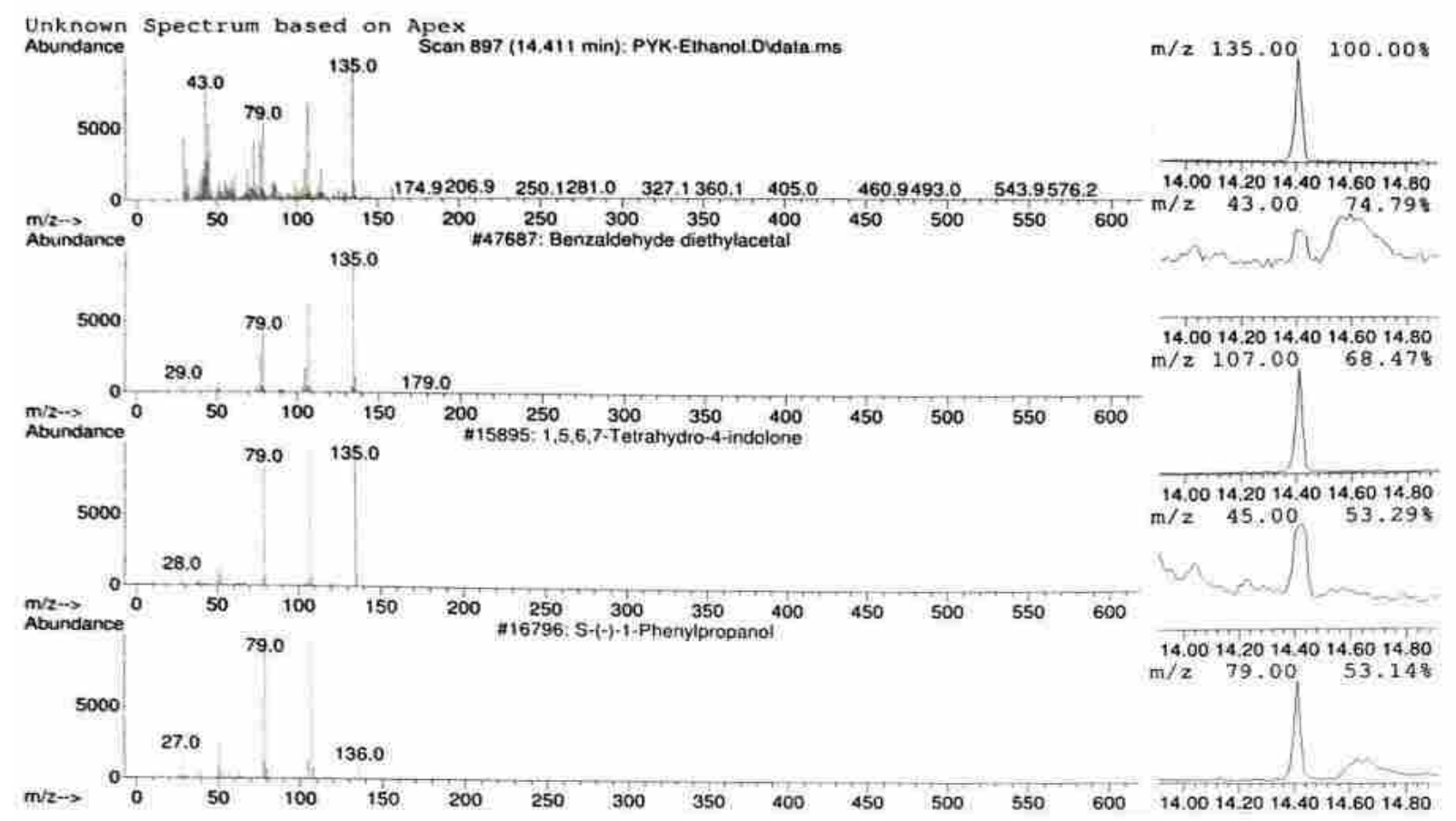

Data File: G: ISarathi documents/Saradhi DocumentslGC-MS DATAl756-PYK-Ethano1.DIPYK-Ethano1.D Sample: 756

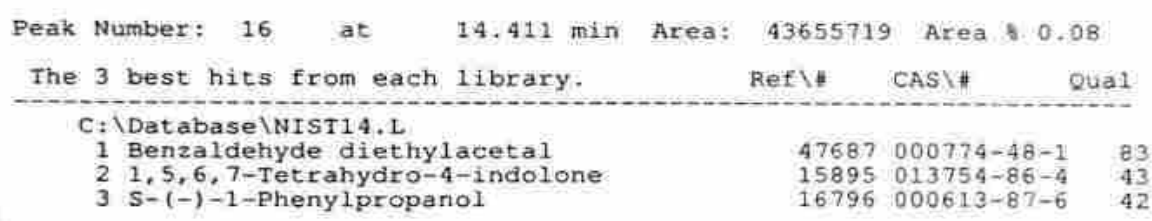<smiles>O=C1CCCc2[nH]ccc21</smiles>

1,5,6,7 tetrahydroindolone<smiles>CC[C@H](O)c1ccccc1</smiles>

S-(-)-1-phenyl propanol 

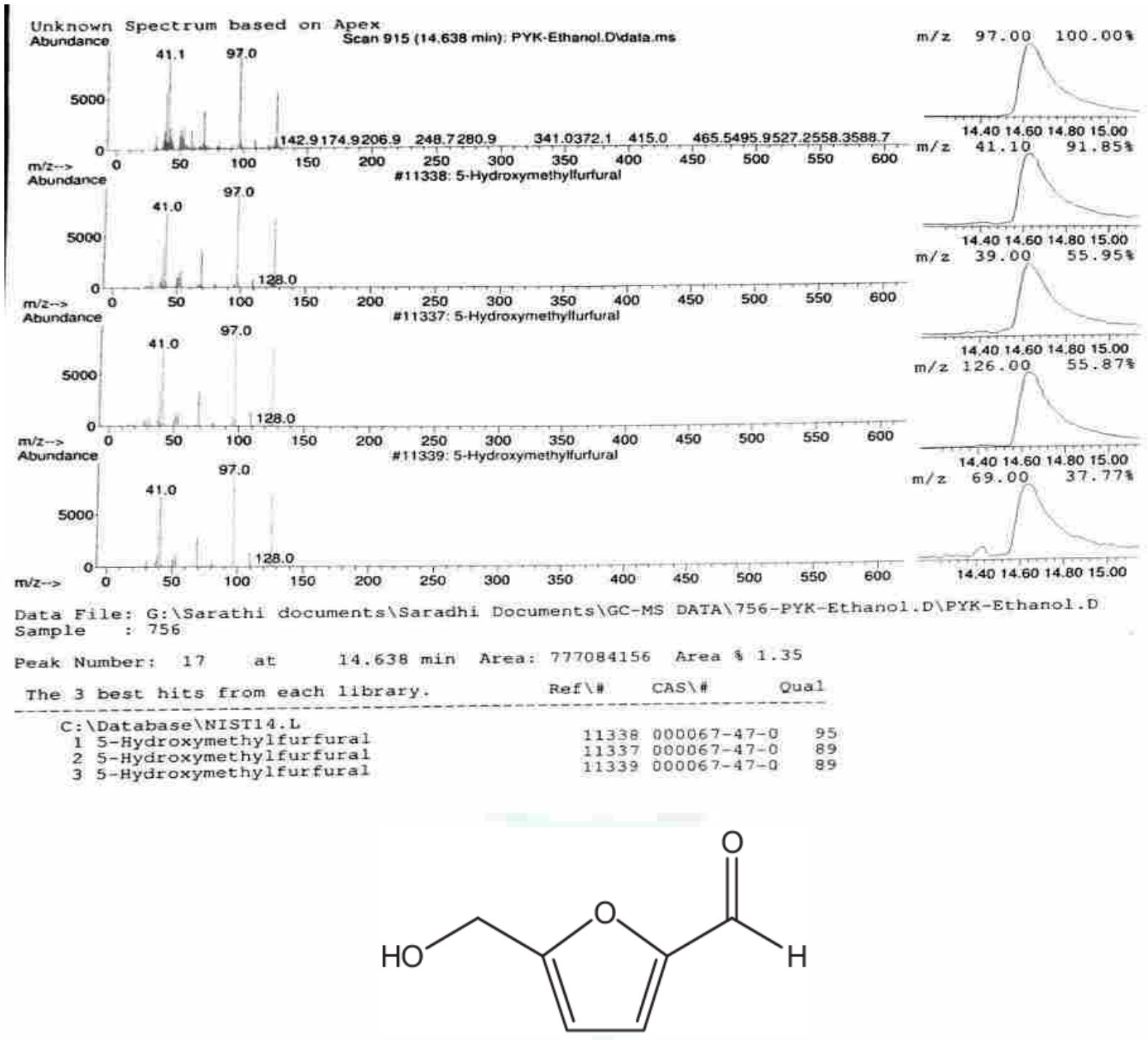

5- Hydroxy methyl furfural 


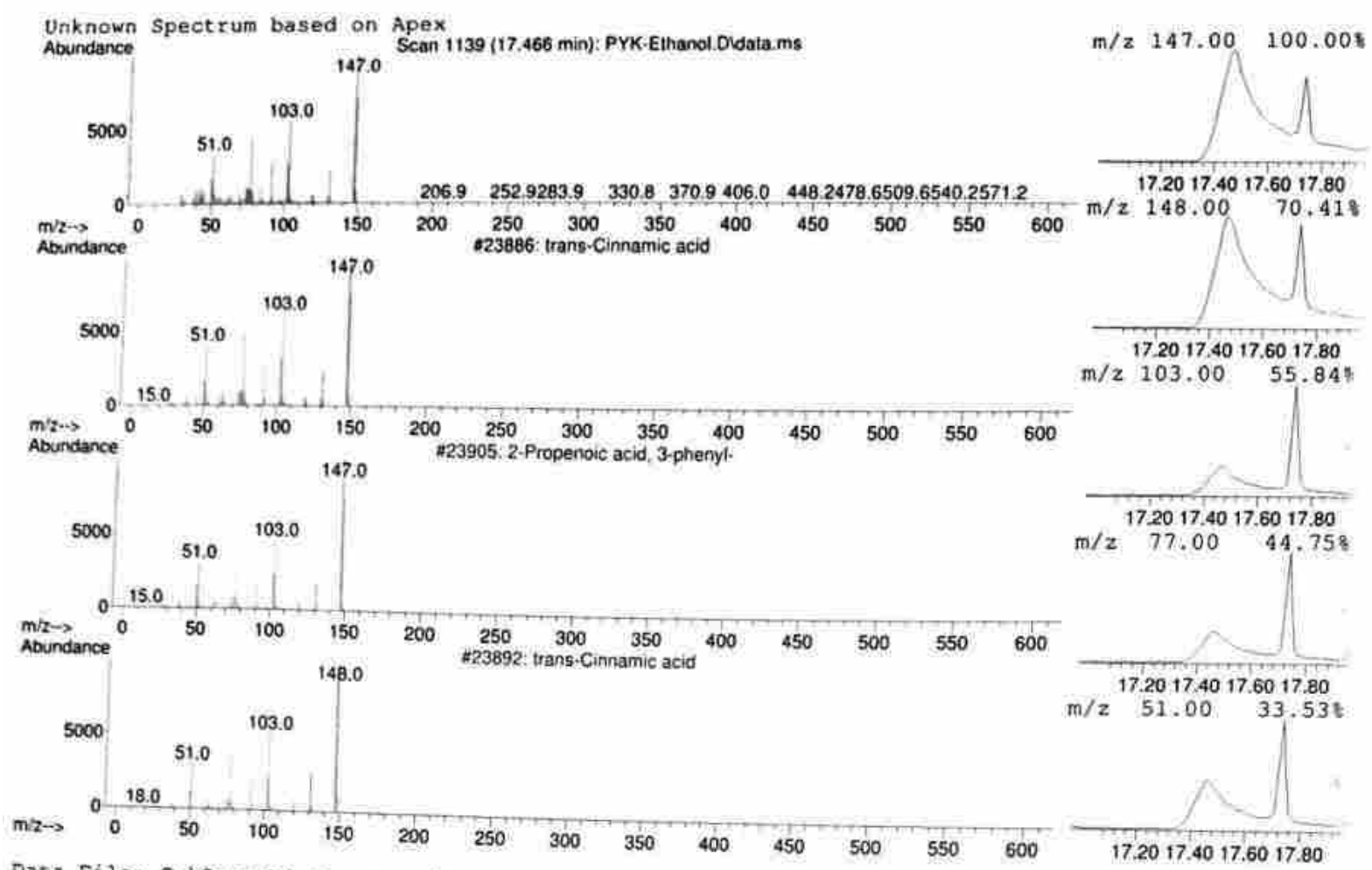

Data File: G: ISarathi documents/Saradhi Documents/GC-MS DATAI756-PYK-Ethano1. D $:$ PYK-Ethanol,D
Sample 756

Peak Number: 20 at $17.466 \mathrm{~min}$ Area: 415870967 Area 0.72

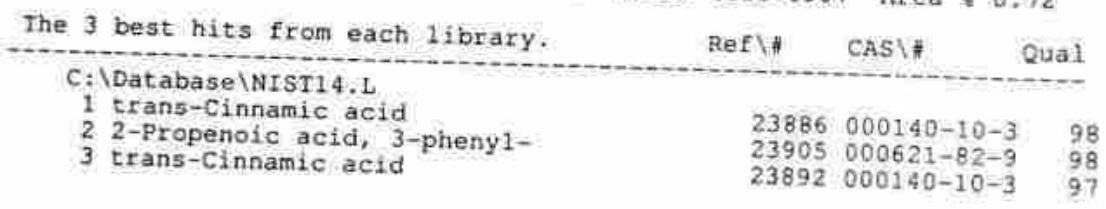<smiles>O=C(O)/C=C/c1ccccc1</smiles><smiles>CCOC(=O)/C=C/c1ccccc1</smiles>

Trans- cinnamic acid

2-propenoic acid, 3-phenyl 


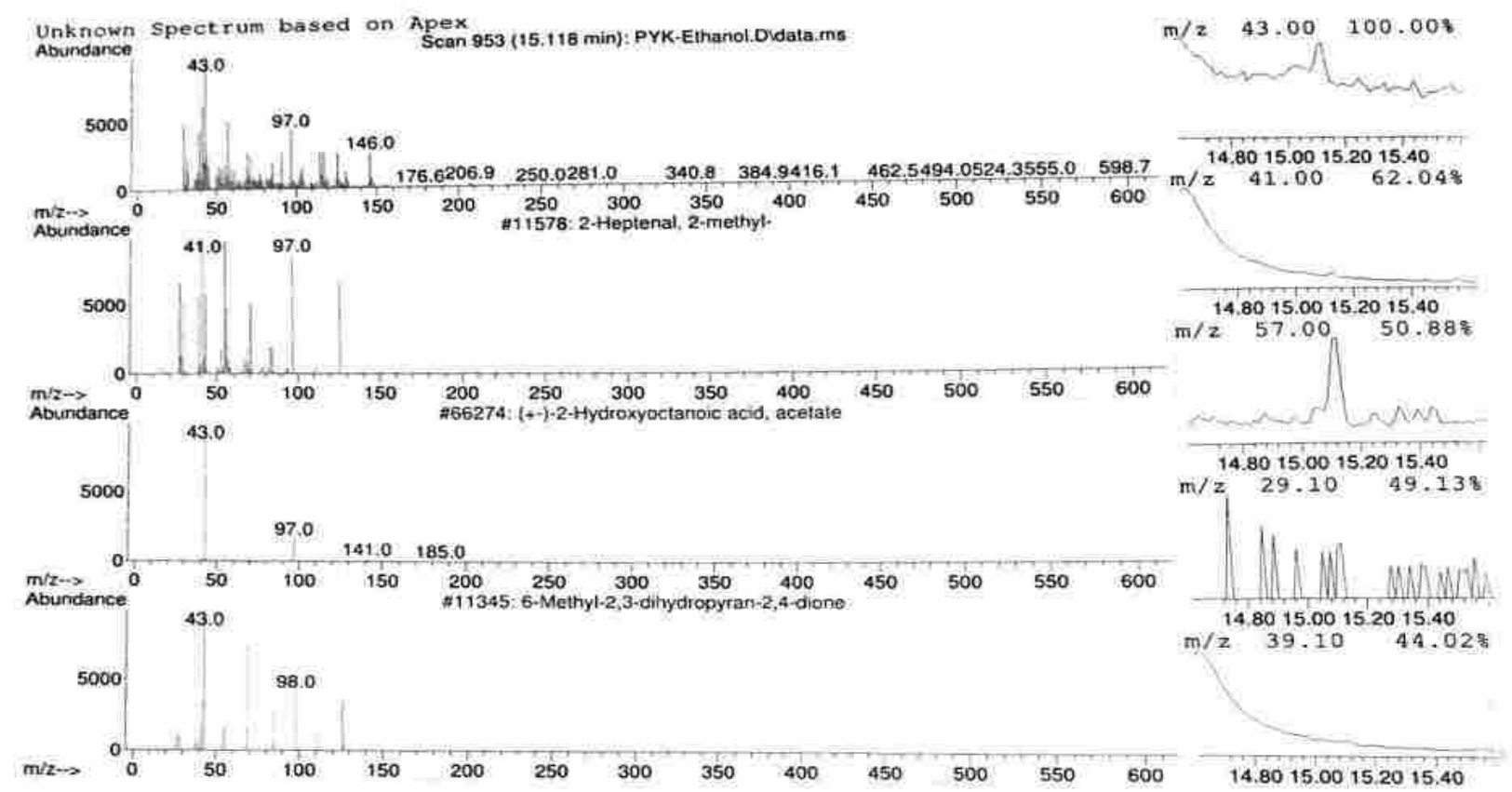
Data File: G: Sarathi documents ISaradhi DocumentslGC-MS DATA 756-PYK-Ethanol. DYPYK-Ethano1.D
Sample : 756

Peak Number: 18 at $15.118 \mathrm{~min}$ Area: 58489723 Area 80.10

The 3 best hits from each library. Refl\# CAS\\# Qua]

C: IDatabase INIST14.L.

1 2-Heptenal, 2-methyl-

2 (+-)-2-Hydroxyoctanoic acid, ace... $\quad 11578030567-26-1 \quad 14$

3 6-Methy1-2,3-dihydropyran-2,4-dione $\quad 11345000541-98-0 \quad 10$

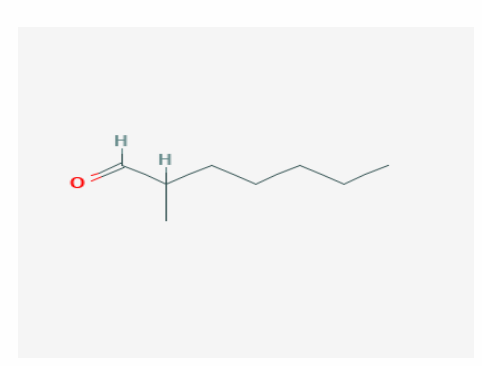

2-hydroxyoctanoic acid

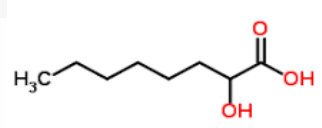

2-heptanal, 2 methyl-<smiles></smiles>

6-methyl-2, 3-dihydropyran-2,4-dione 


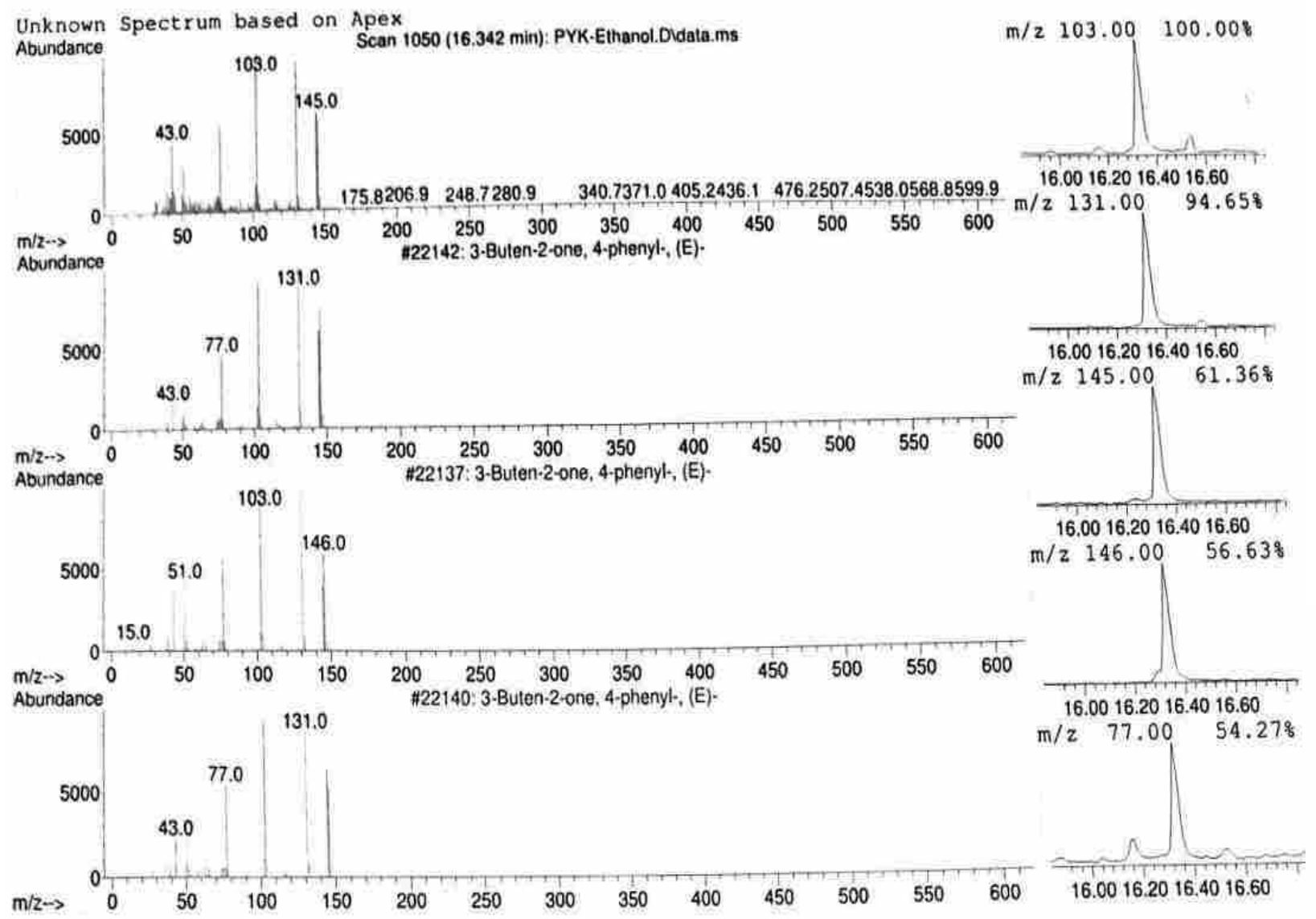

Data File: G: \Sarathi documents\Saradhi Documents\GC-MS DATA 756 -PYK-Ethanol.D \PYK-Ethanol.D Sample : 756

Peak Number: 19 at $16.342 \mathrm{~min}$ Area: 61390636 Area 80.11

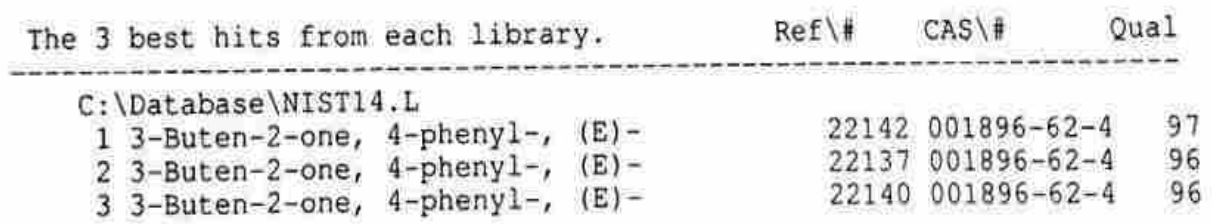

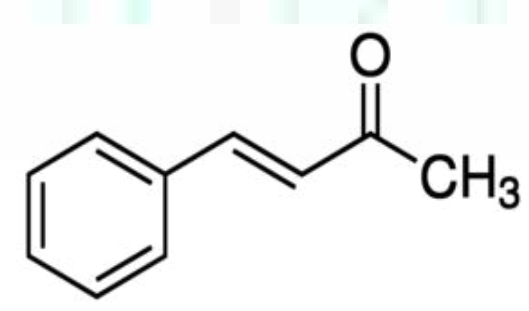

3-buten-2-one, 4-phenyl 


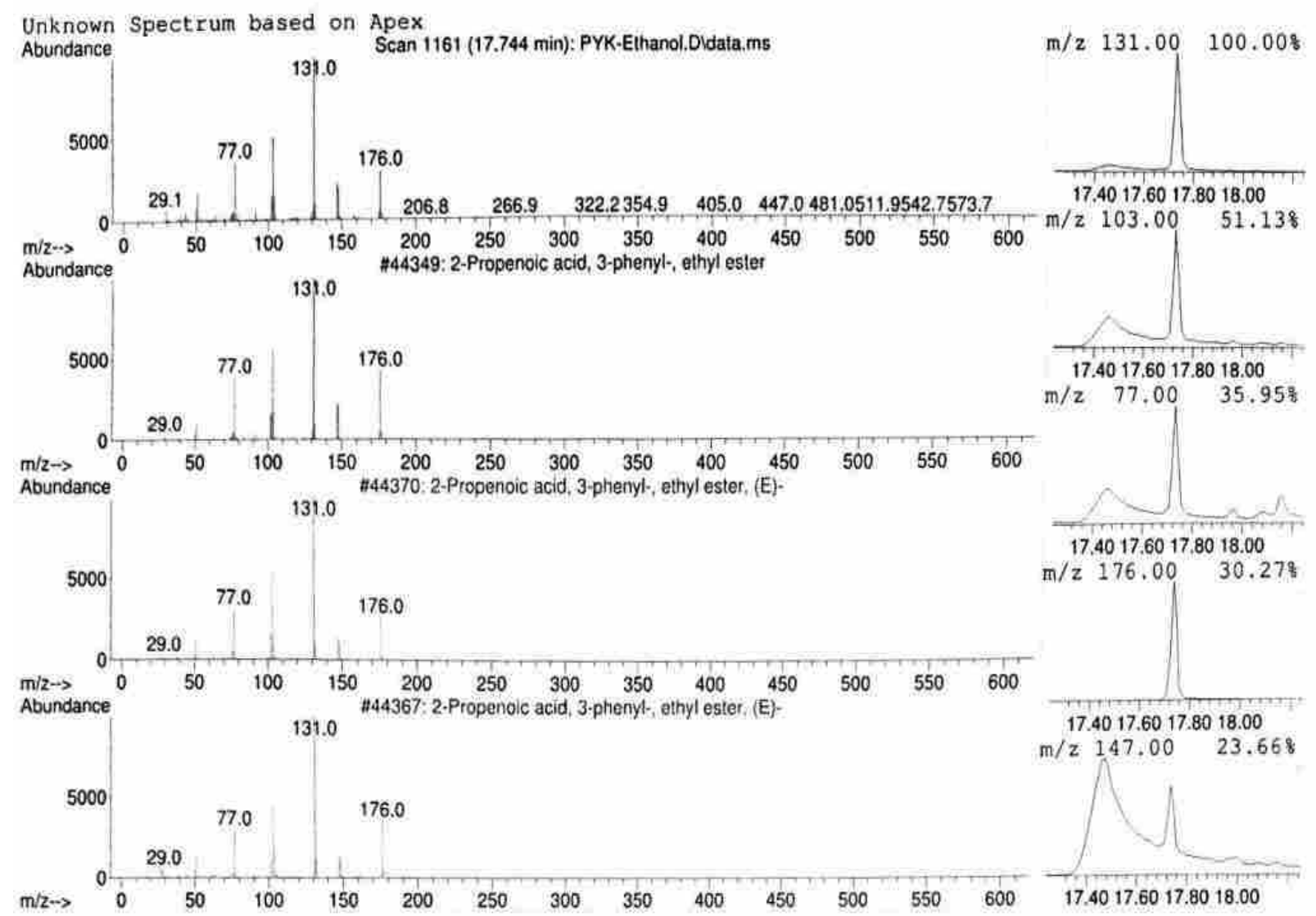

Data File: G: \Sarathi documents\Saradhi Documents\GC-MS DATA IT56-PYK-Ethano1.D\PYK-Ethanol.D Sample: 756

Peak Number: 21 at $17.744 \mathrm{~min}$ Area: 301479569 Area 0.52

The 3 best hits from each library. Ref $\backslash \# \quad C A S \backslash \# \quad$ Qual

C: Database INIST14.L

1 2-Propenoic acid, 3-phenyl-, eth...

$44349000103-36-6 \quad 98$

3 2-Propenoic acid, 3-phenyl-, eth... $44367004192-77-2 \quad 94$

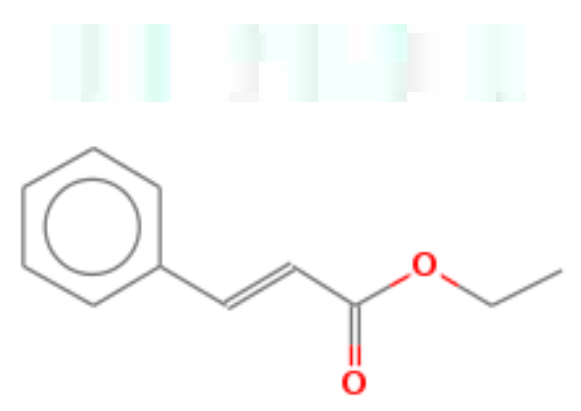

2-propenoic acid, 3-phenyl-ethyl ester 


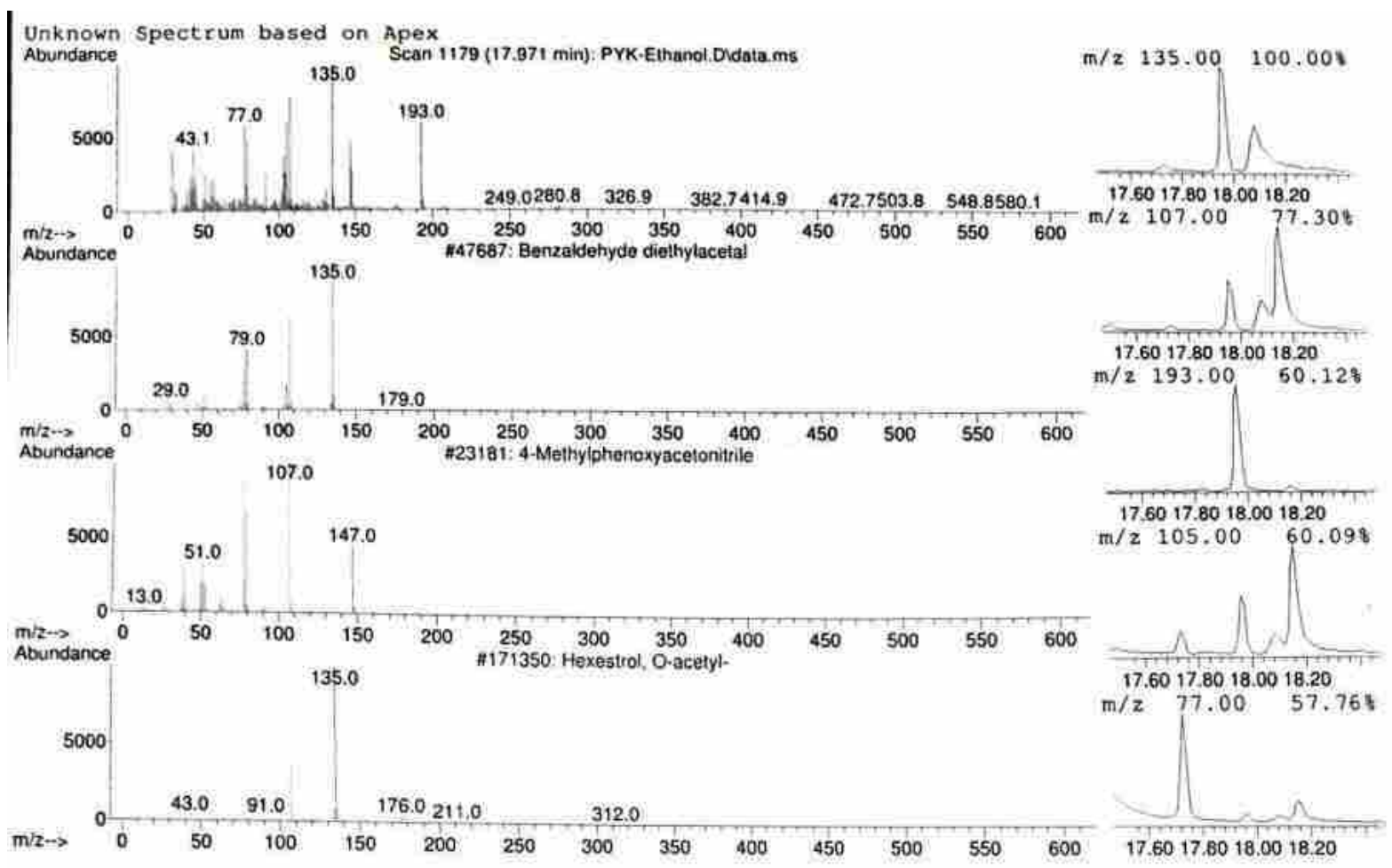
Data File: G: ISarathi documentslSaradhi DocumentslGC-MS DATAl756-PYK-Ethanol.DVPYK-Ethanol.D
Sample : 756

Peak Number: 22 at $17.971 \mathrm{~min}$ Area: 54551820 Area $\$ 0.09$

The 3 best hits from each library. Refl\# CASI\# oual

C: \Database INIST14.

1 Benzaldehyde diethylacetal

2 4-Methylphenoxyacetonitrile

3 Hexestrol, 0-acetyl-

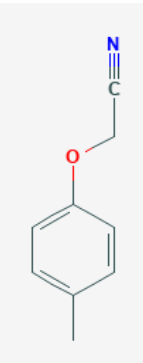

\section{4-methyl phenoxy acetonitrile}

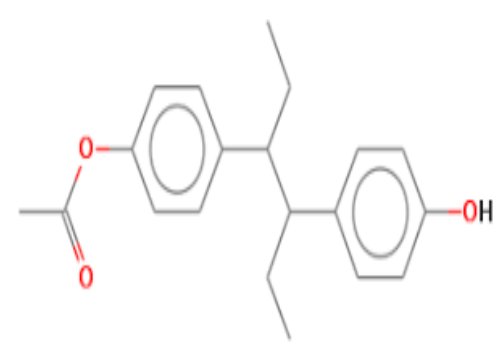

hexestrol, o-acetyl 


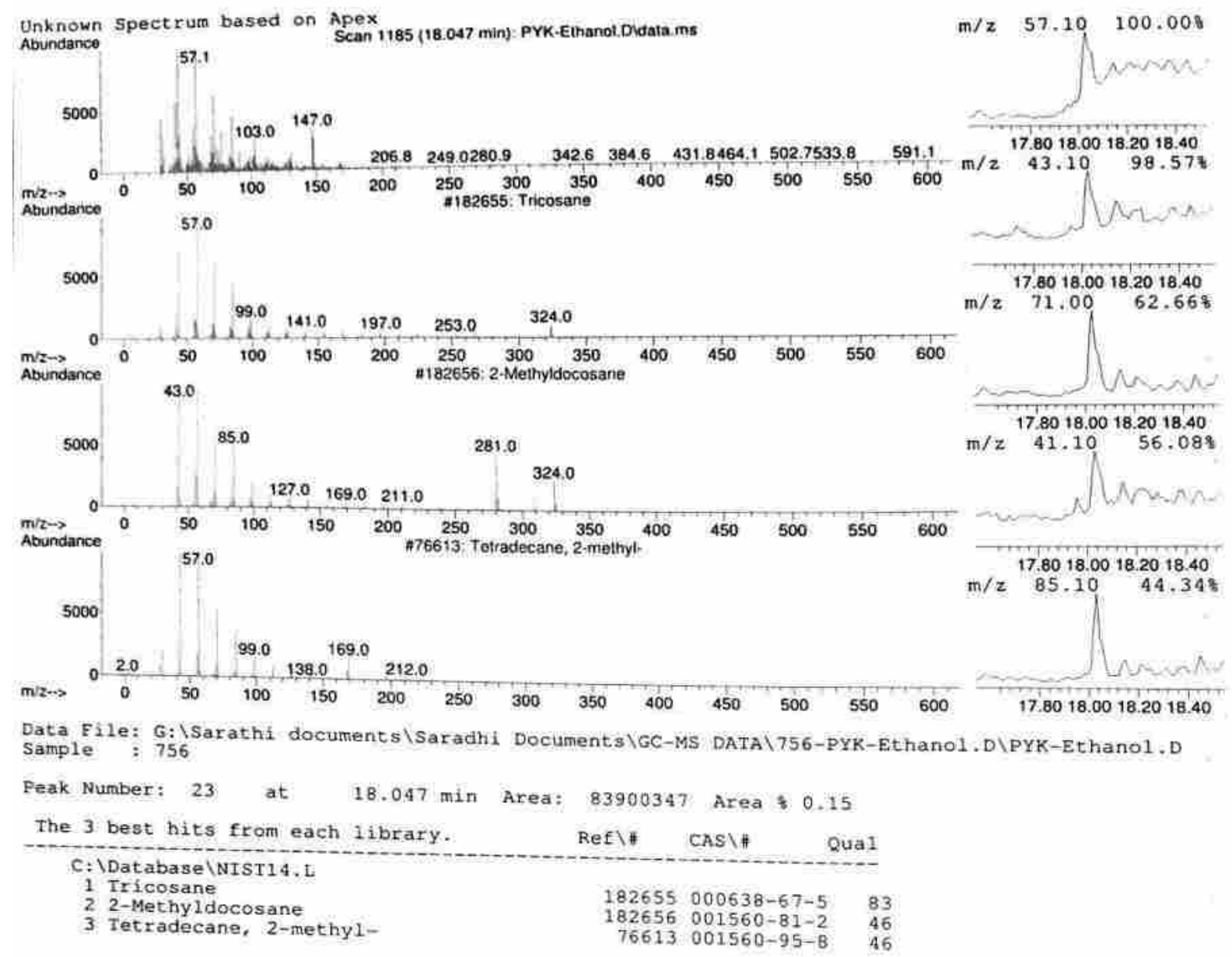

Tricosane

2-methyl docosane

Tetradecane, 2 methyl- 

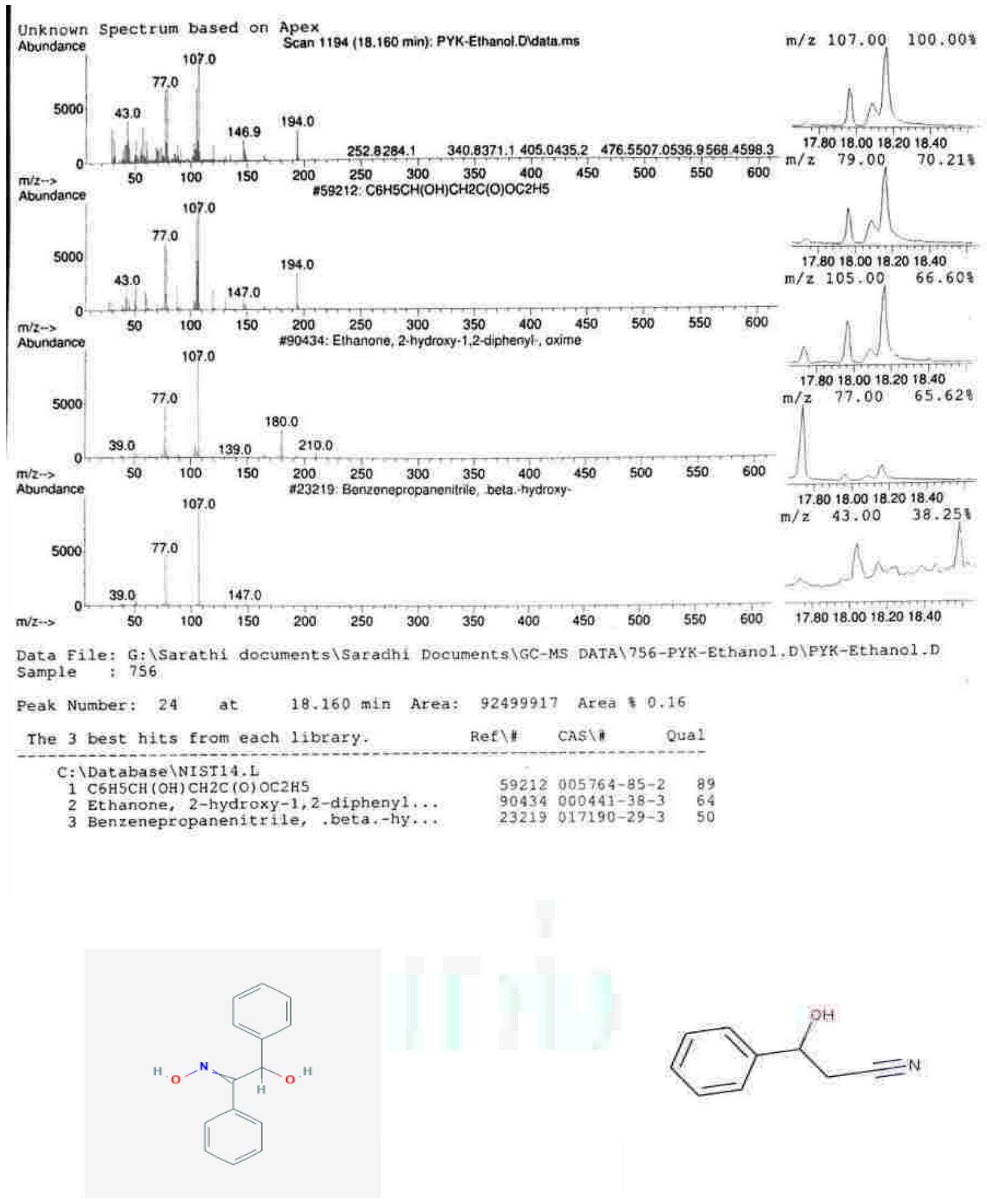

Ethanone , 2-hydroxy-1,2-diphenyl,oxime

Benzenepropanenitrile, beta hydroxy 


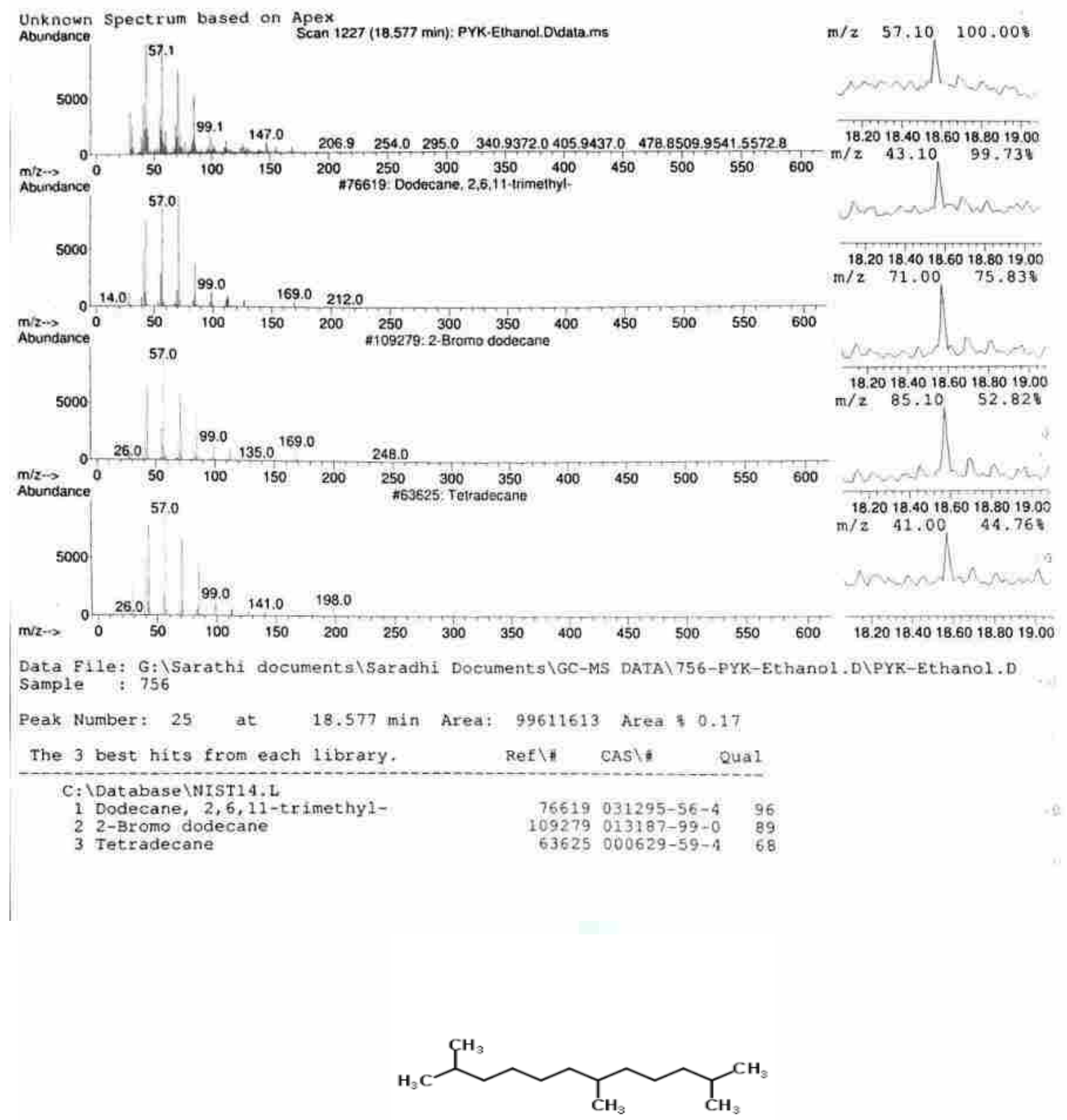

Dodecane, 2,6,11 -tri methyl- 


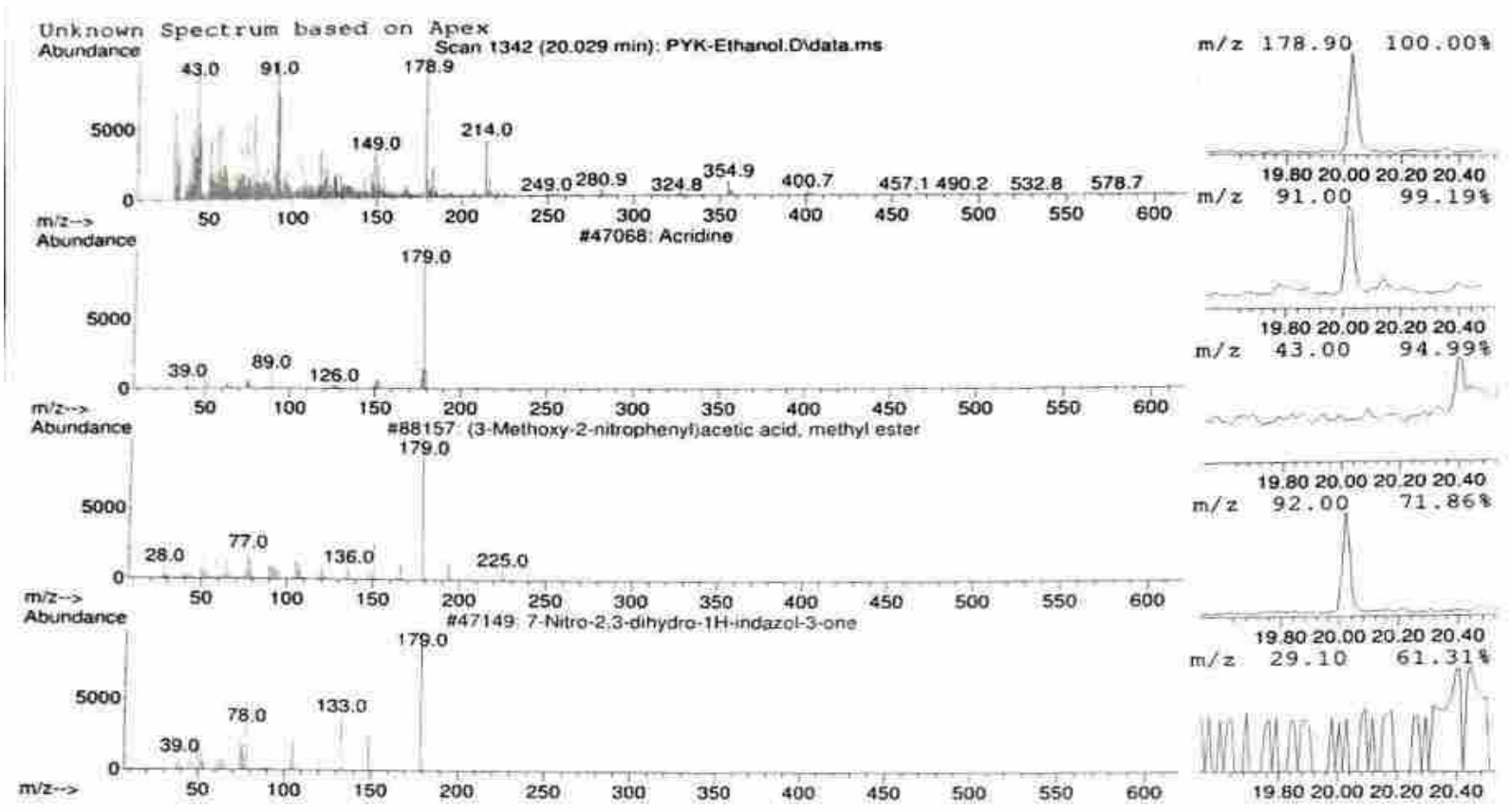

Data File: G: ISarathi documents SSaradhi DocumentsIGC-MS DATAI756-PYK-Ethanol.DIPYK-Ethanol.D Sample: 756

Peak Number: 27 at $20.029 \mathrm{~min}$ Area: 22017138 Area 0.04

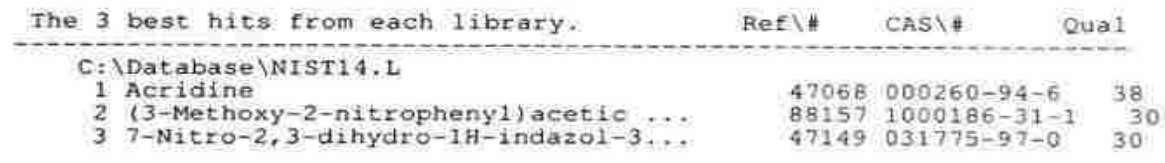

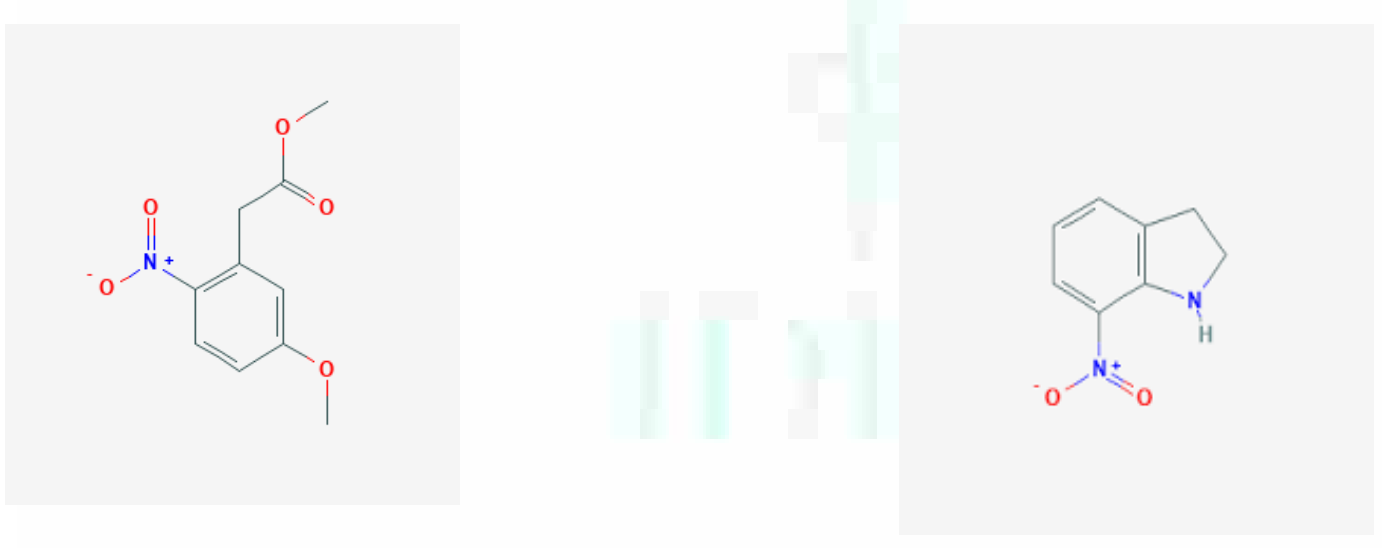

(3-methoxy-2-nitrophenyl)aceticacid, 7-nitro 2,3-dihydro-1H-indazole-3-one Methyl ester 


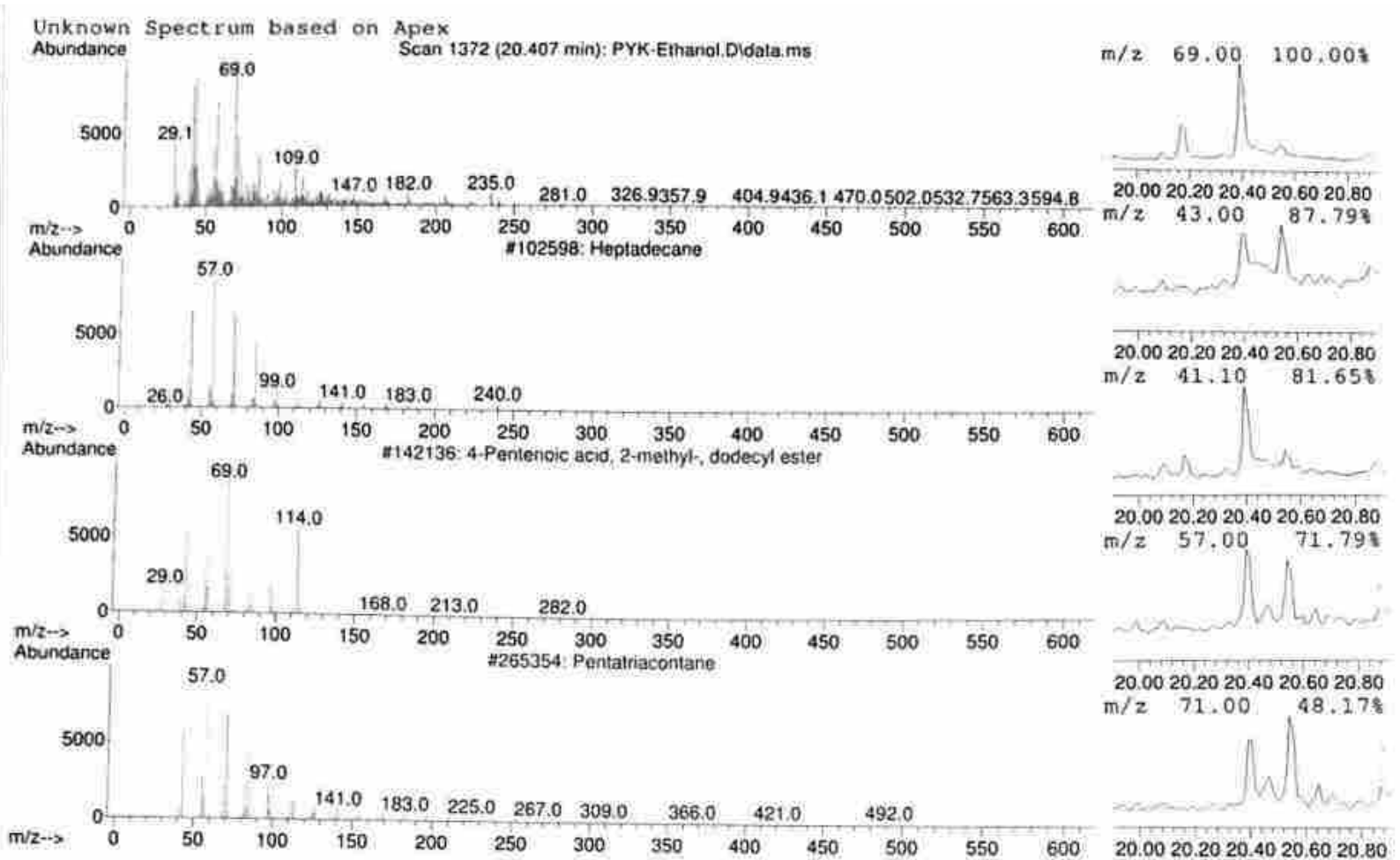
Data File: G: ISarathi documentslSaradhi DocumentslGC-MS DATA $756-$ PYK-Ethano1.DVPYK-Ethano1.D
Sample : 756

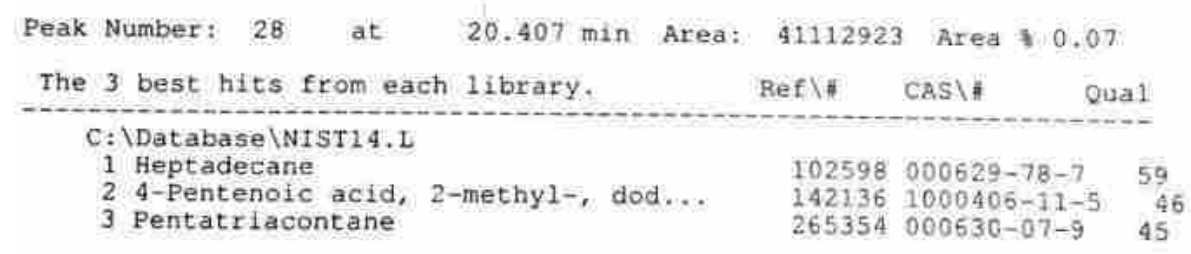

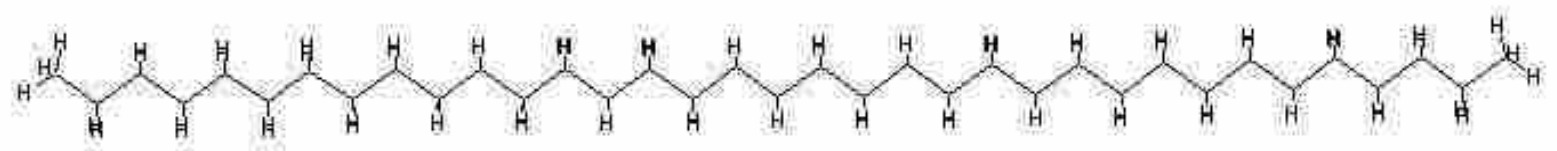

Pentatriacontane 


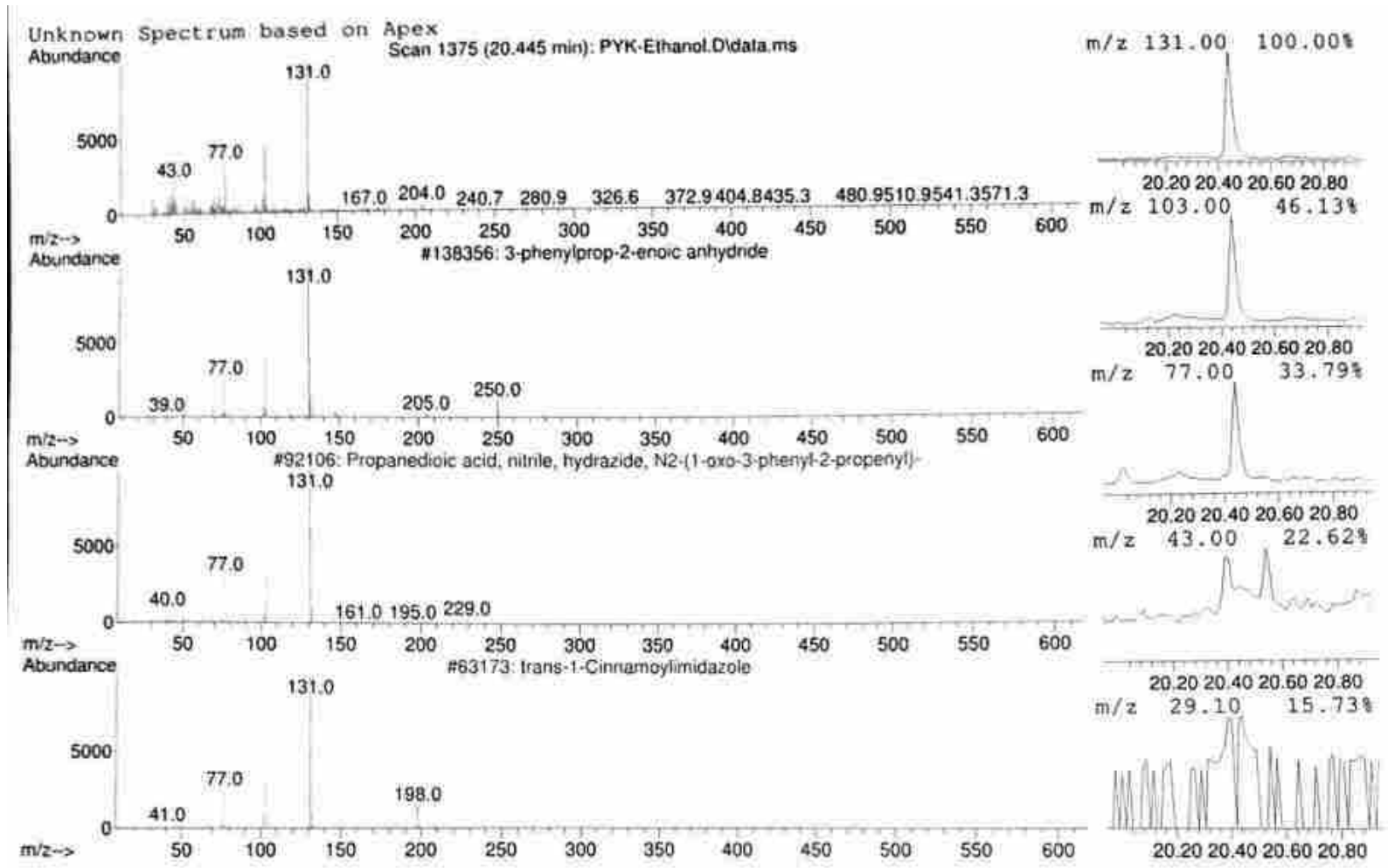

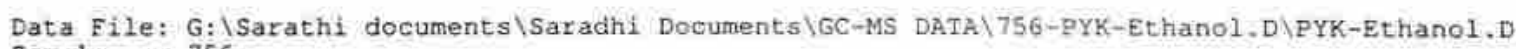
Sample : 756

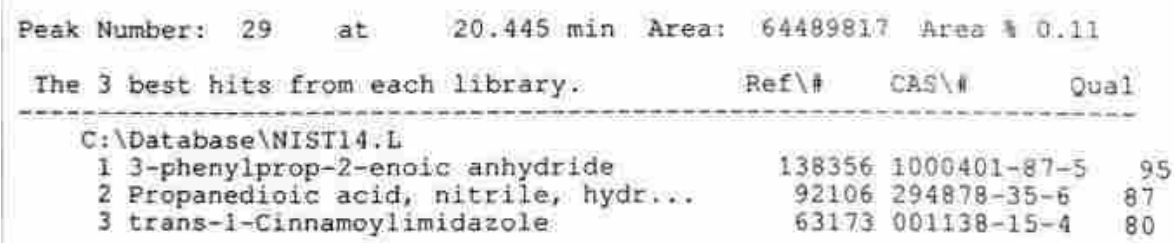

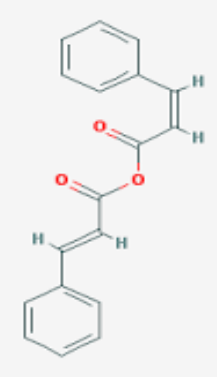

trans-1-cinnamoylimidazole<smiles>O=C(/C=C\c1ccccc1)n1ccnc1</smiles> 


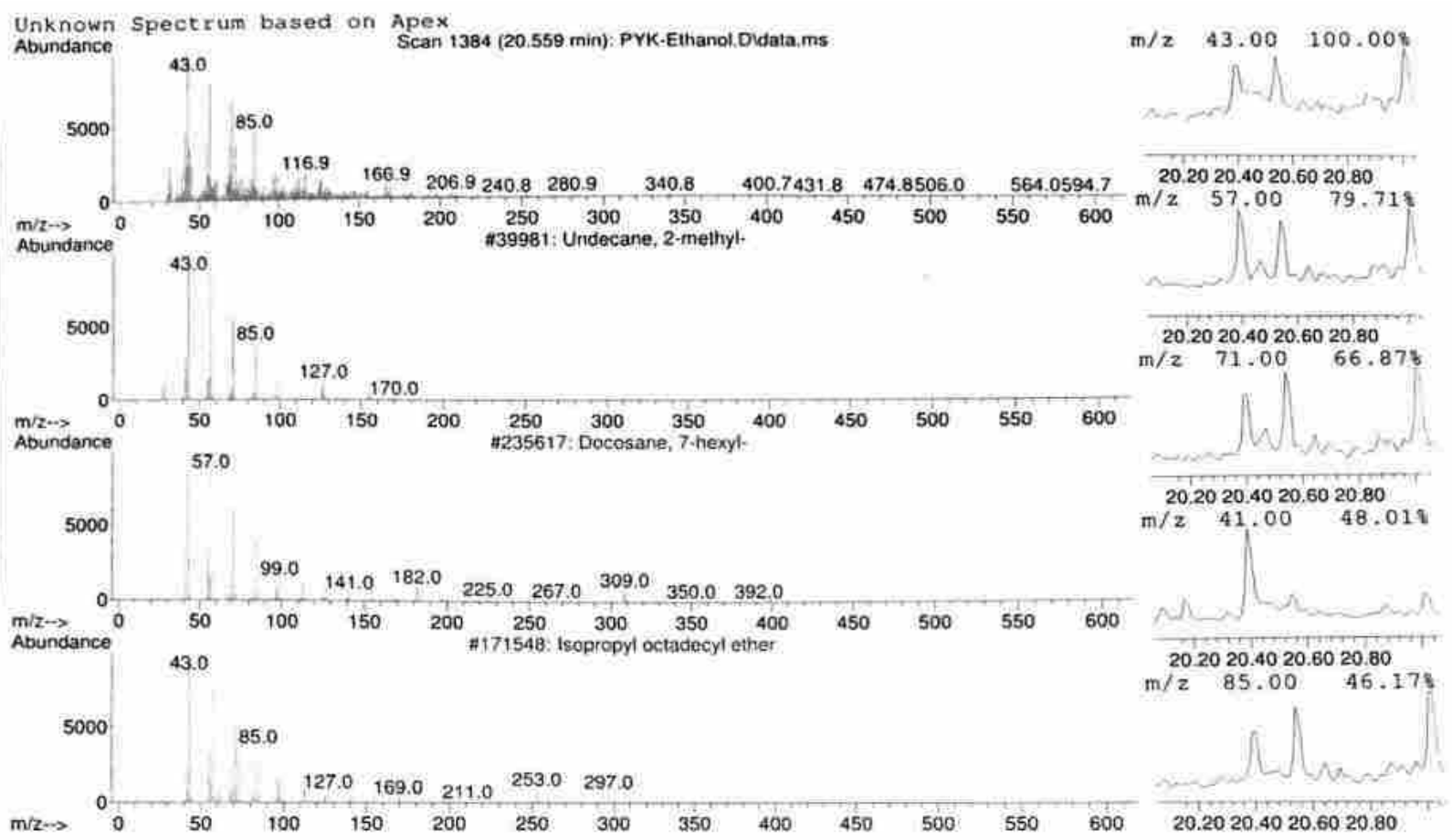

Data File: G: ISarathi documents ISaradhi DocumentslGC-MS DATAl756-PYK-Ethanol.DIPYK-Ethano1.D Sample: 756

Peak Number: 30 at $20.559 \mathrm{~min}$ Area: 23611192 Area $\$ 0.04$

The 3 best hits from each 1 ibrary. ReEY\# CASI\# Qua1

C: \DatabaselNIST1 4.L.

1 Undecane, 2-methyl-

2 Docosane, 7-hexyi-

3 Isopropyl octadecyl ether

39981 007045-71-8 $\quad 90$

$\begin{array}{lll}235617 & 055373-86-9 & 83 \\ 171548 & 1000406-34-2\end{array}$

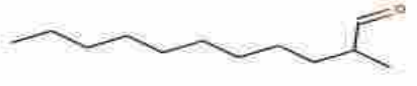

Undecane, 2-methyl

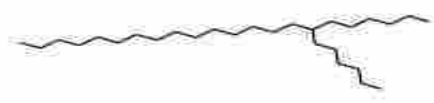

dosocane, 7-hexyl-

Isopropyl octadecyl ether 


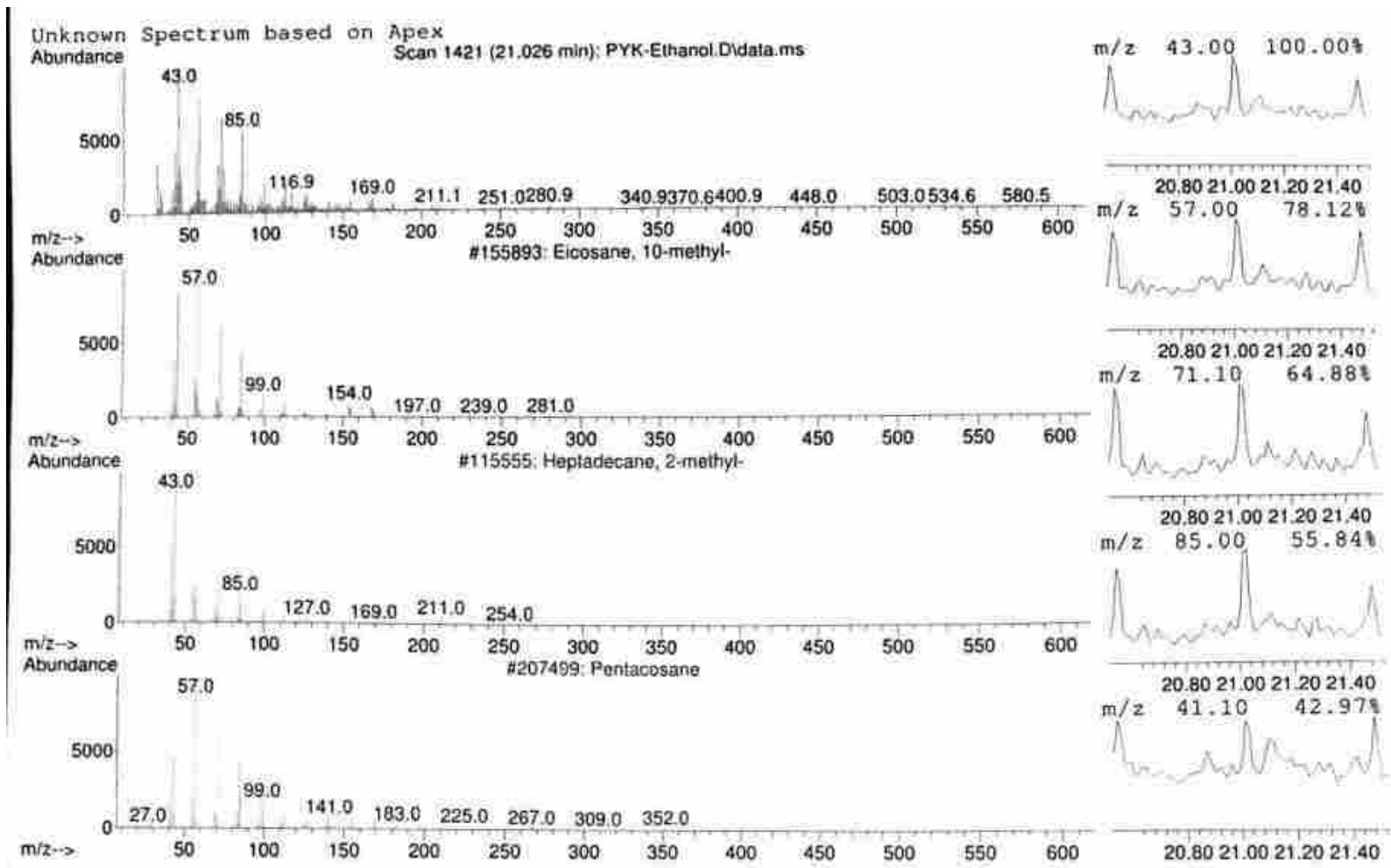

Data File: G: ISarathi documents Saradhi Documents VGC-MS DATAI756-PYK-Ethanol.DVPYK-Ethanol.D Sample: 756

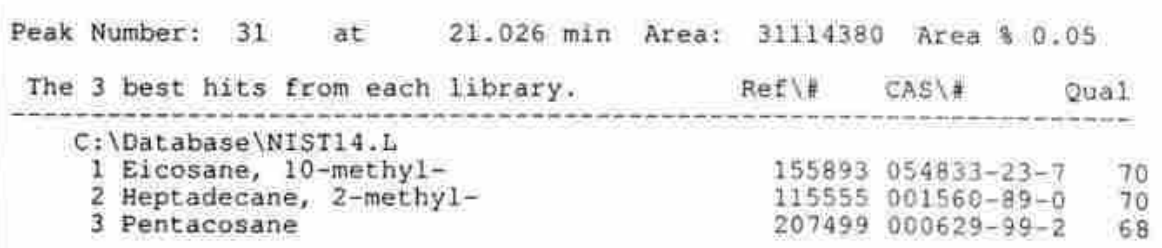




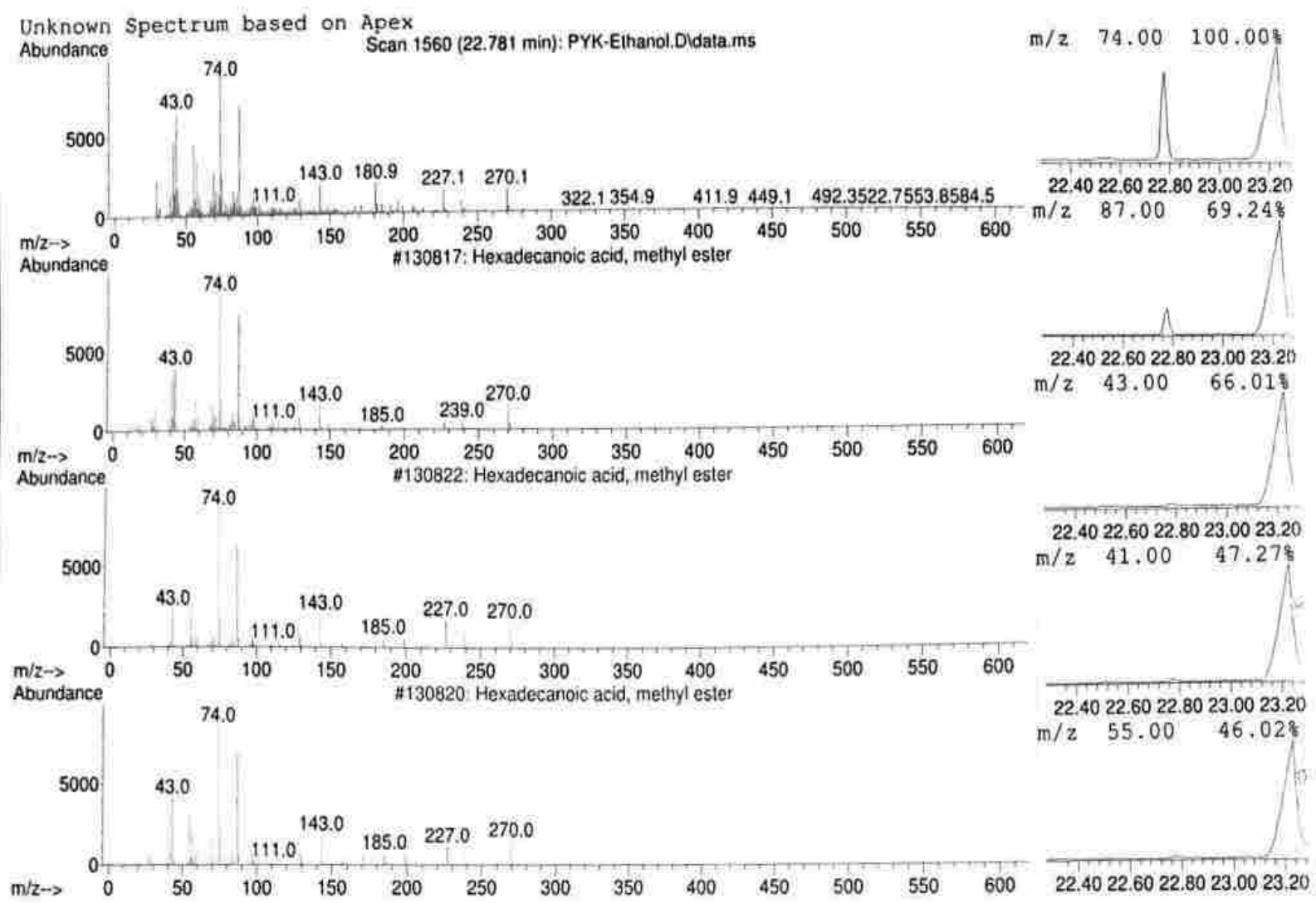

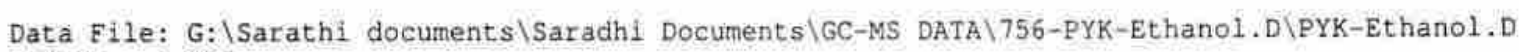
Sample : 756

Peak Number: 32 at $22.781 \mathrm{~min}$ Area: 46346808 Area $\$ 0.08$

\begin{tabular}{llll} 
The 3 best hits from each library. & Ref $\backslash \#$ & CAS $\backslash \#$ & Qual \\
\hline C: $:$ DatabaselNIST14.L & & & \\
1 Hexadecanoic acid, methy1 ester & 130817 & $000112-39-0$ & 98 \\
2 Hexadecanoic acid, methyl ester & 130822 & $000112-39-0$ & 97 \\
3 Hexadecanoic acid, methyl ester & $130820000112-39-0$ & 96
\end{tabular}

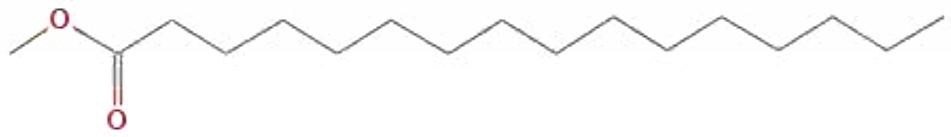

Hexadecenoic acid, methyl ester 


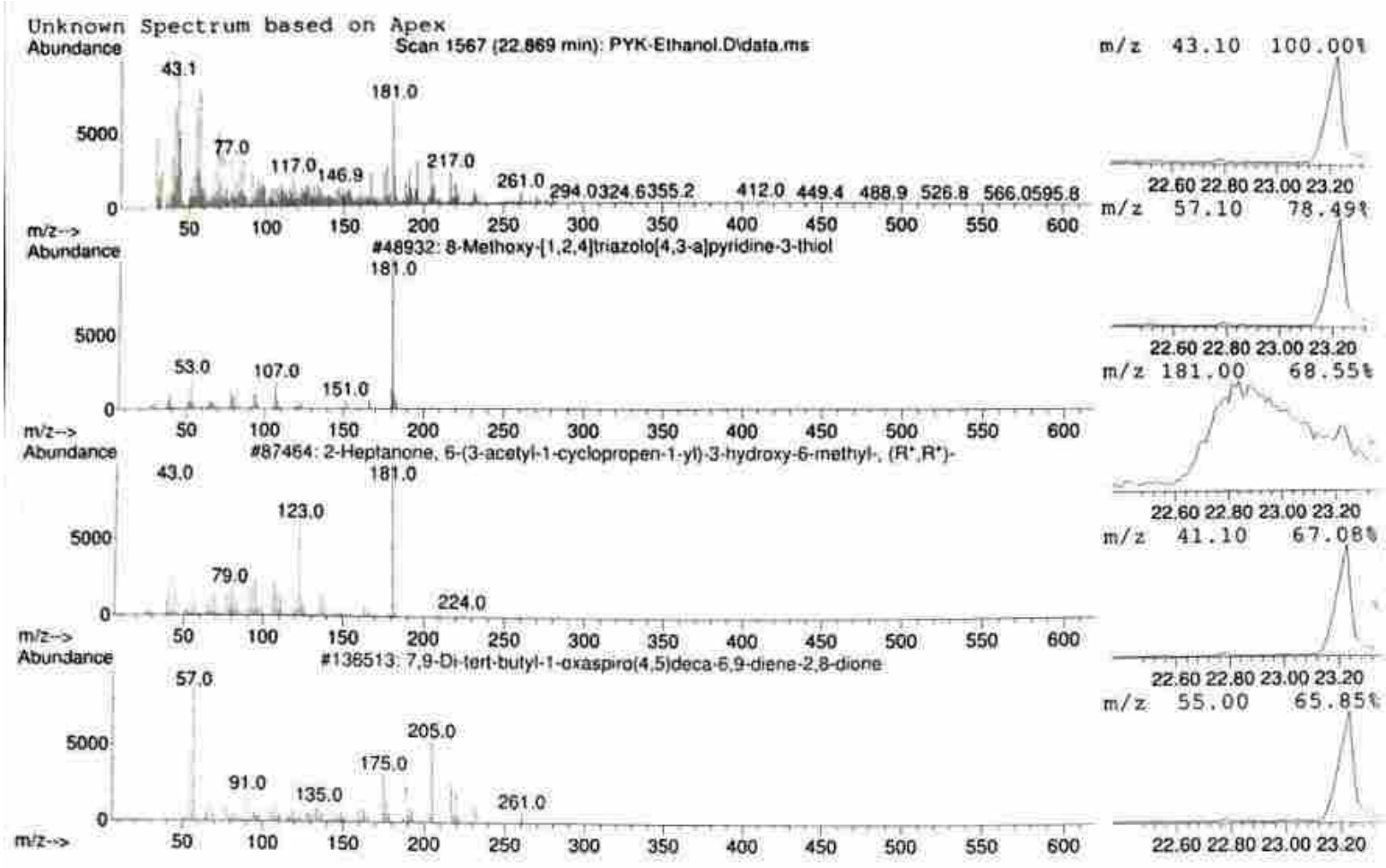

Data File: G: ISarathi documents \Saradhi Documents\GC-MS DATA 756-PYK-Ethano1.D\PYK-Ethano1.D Sample: 756

Peak Number: 33 at $22.869 \mathrm{~min}$ Area: 18906830 Area 10.03

The 3 best hits from each Iibrary. Refl\# CASl"

C: IDatabase INIST14, L

1 8-Methoxy-11,2,4] triazolo[4,3-a]... $489321000186-80-8 \quad 53$

2 2-Heptanone, 6-13-acety1-1-cyclo... $\quad 87464078091-85-7 \quad 53$

37,9 -Di-tert-buty $1-1$-oxaspiro $14,5 \ldots \quad 136513 \quad 082304-66-3 \quad 50$

7,9-di-tert-butyl-1-oxaspiro(4,5)deca-5,9diene-2,8-dione 


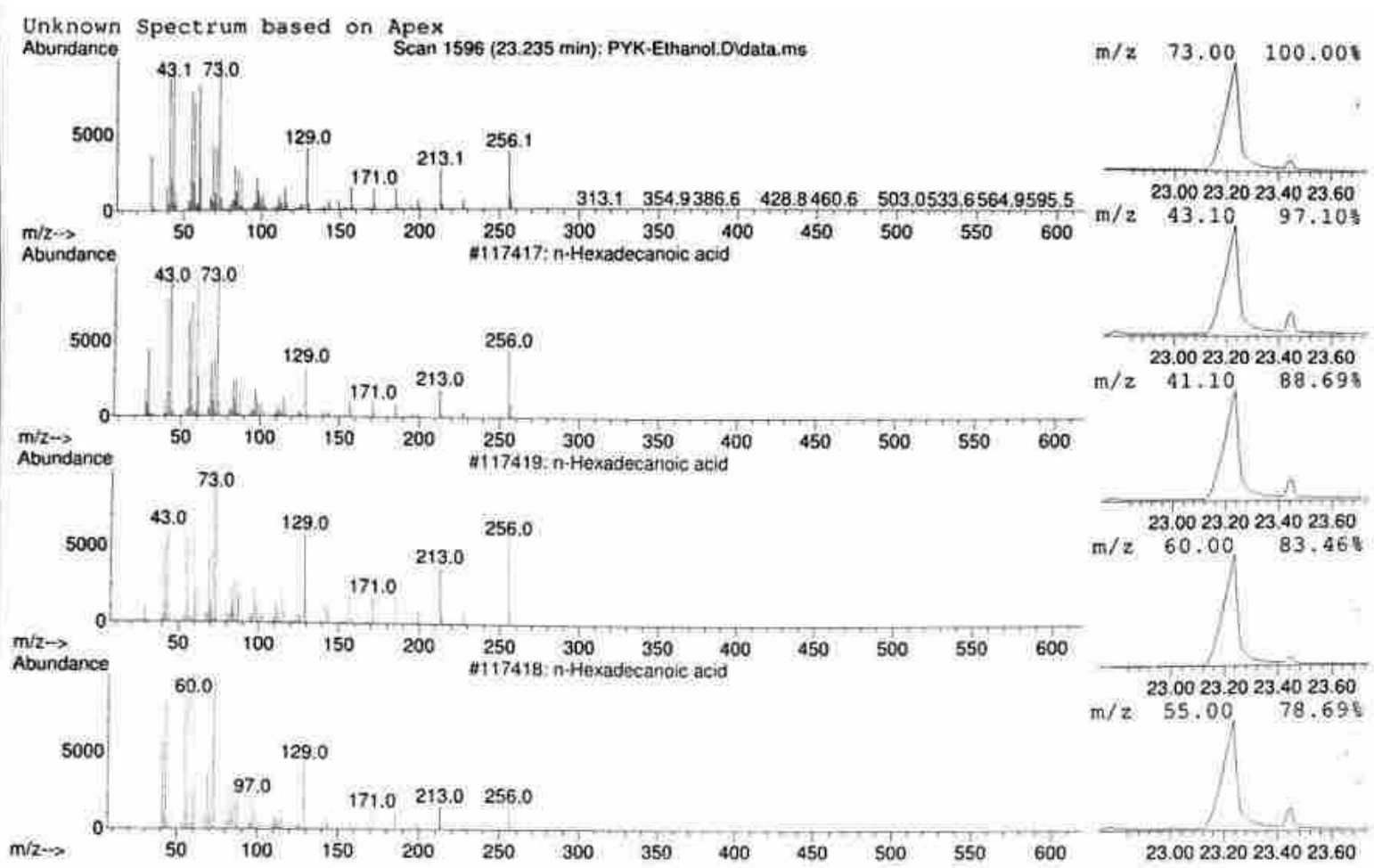

Data File: G: \Sarathi documentslSaradhi DocumentsiGC-MS DAIA 756 -PYK-Ethano1.D D PYK-Ethanol.D Sample : 756

Peak Number: 34 at $23.235 \mathrm{~min}$ Area: 2686327858 Area $\$ 4.65$

The 3 best hits froth each library. ReflF CASTF Qua1

C: IDatabaselNIST1 4.

1 n-hexadecanoic acid

2 n-Hexadecanoic acid

3 n-Hexadecanoic acid

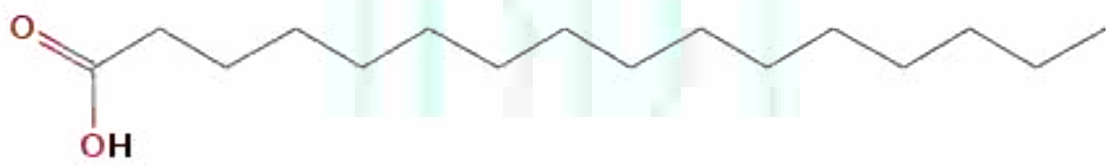

n-Hexadecanoic acid 


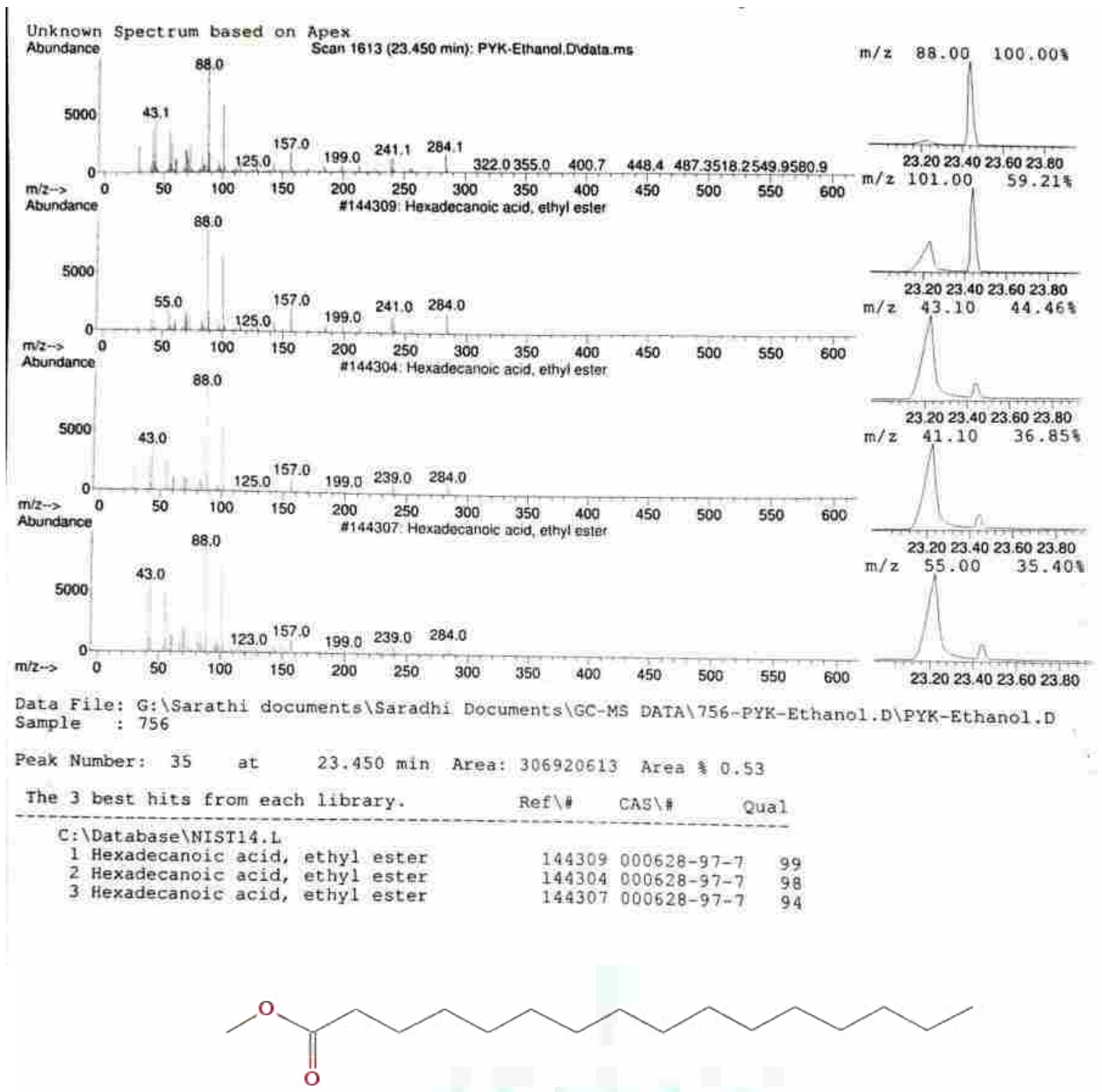

Hexadecenoic acid, methyl ester 


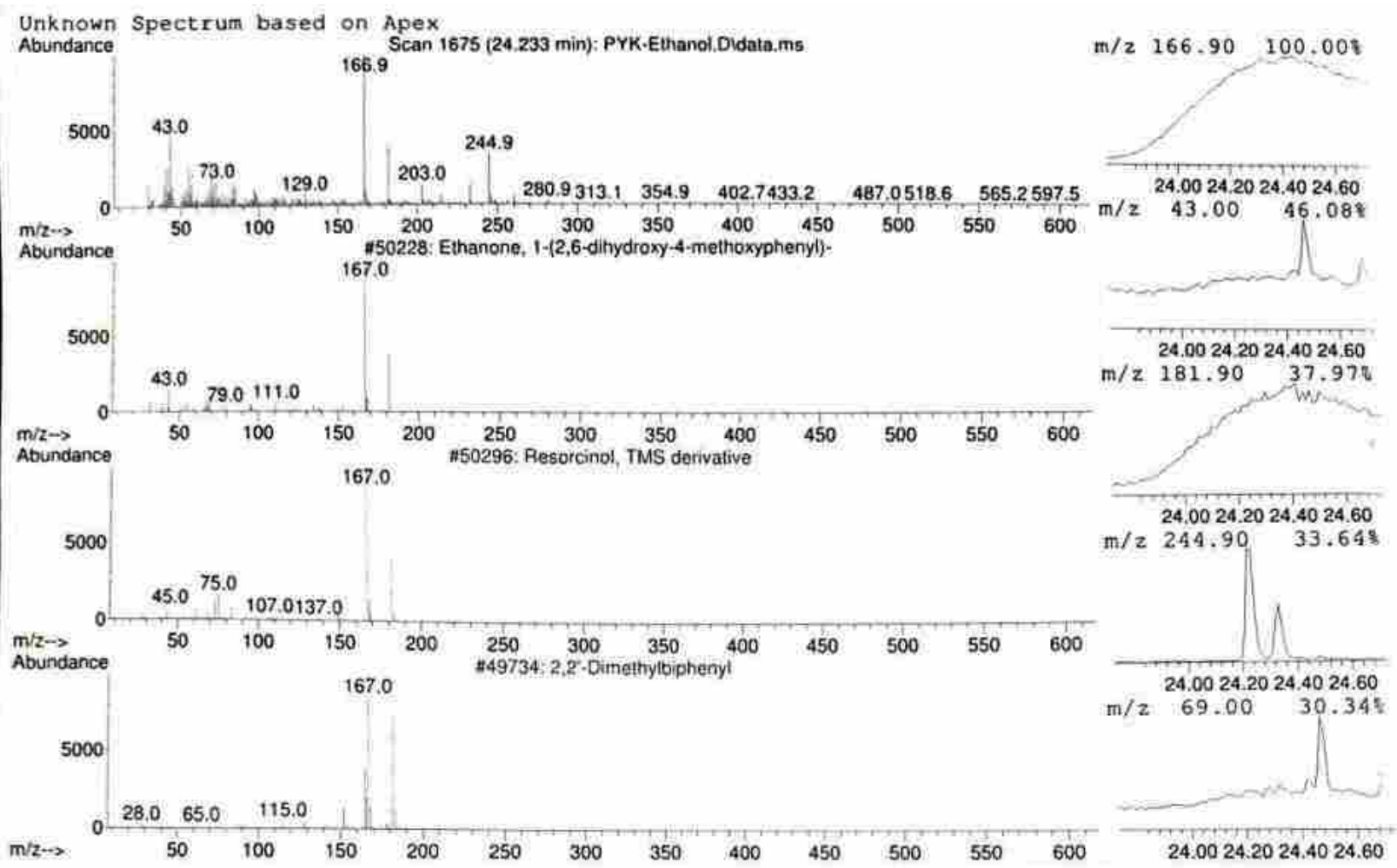

Data File: G: \Sarathi documents\Saradhi Documents\GC-MS DATA 756 -PYK-Ethanol.D\PYK-Ethano1.D Sample : 756

Peak Number: 36 at $24.233 \mathrm{~min}$ Area: 75660721 Area 0.13

The 3 best hits from each library. Ref\\# CAS\\# Qual

$\begin{array}{lllll}\text { C: lDatabaselNIST14.L } & & & & \\ 1 \text { Ethanone, 1- } 2,6 \text {-dihydroxy-4-met... } & 50228 & 007507-89-3 & 91 \\ 2 \text { Resorcinol. TMS derivative } & 50296017882-01-8 & 53\end{array}$

$32,2^{\circ}$-Dimethylbipheny1 $\quad 49734000605-39-0 \quad 49$
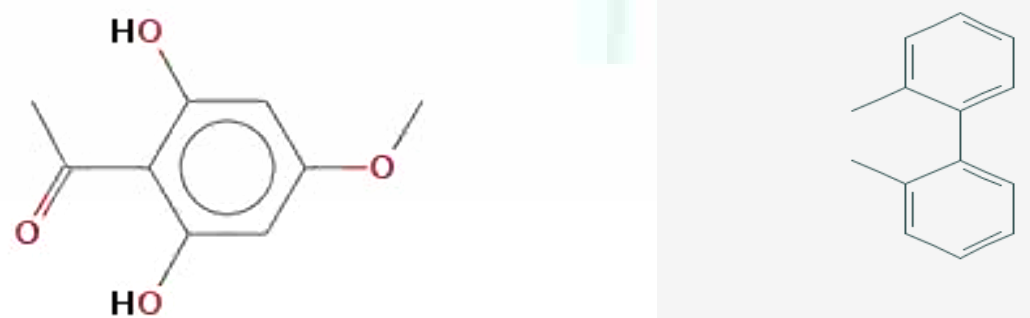

Ethenone, 1-(2, 6-dihydroxy-4-methoxyphenyl)-

2,2'-Dimethylbiphenyl 


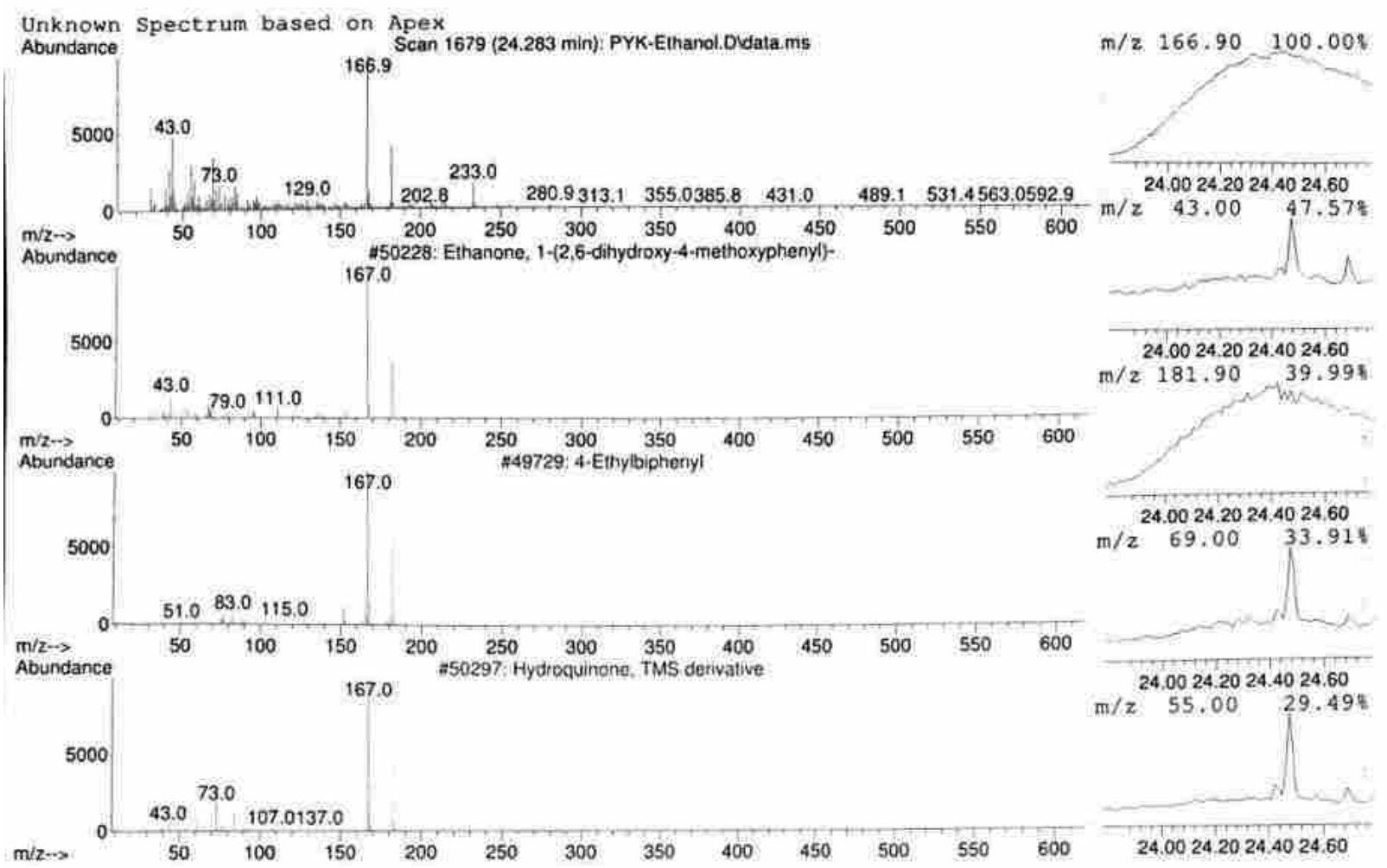

Data File: G: ISarathi documents\Saradhi Documents\GC-MS DATA 756-PYK-Ethano1.DIPYK-Ethano1.D

Sample : 756

Peak Number: 37 at $24.283 \mathrm{~min}$ Area: 18602553 Area $\$ 0.03$

The 3 best hits from each library. Refl\# CAS \# Qual

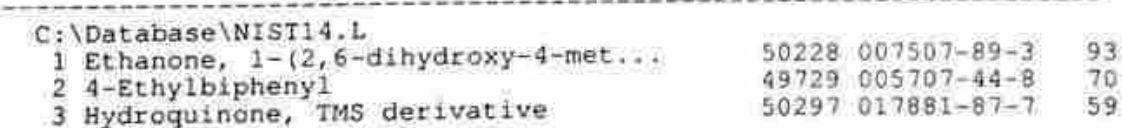
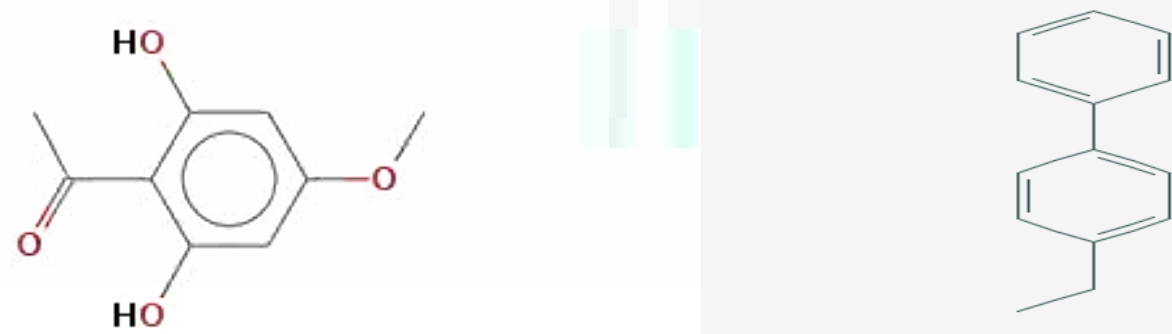

Ethenone, 1-(2, 6-dihydroxy-4-methoxyphenyl)-4-Ethylbiphenyl 

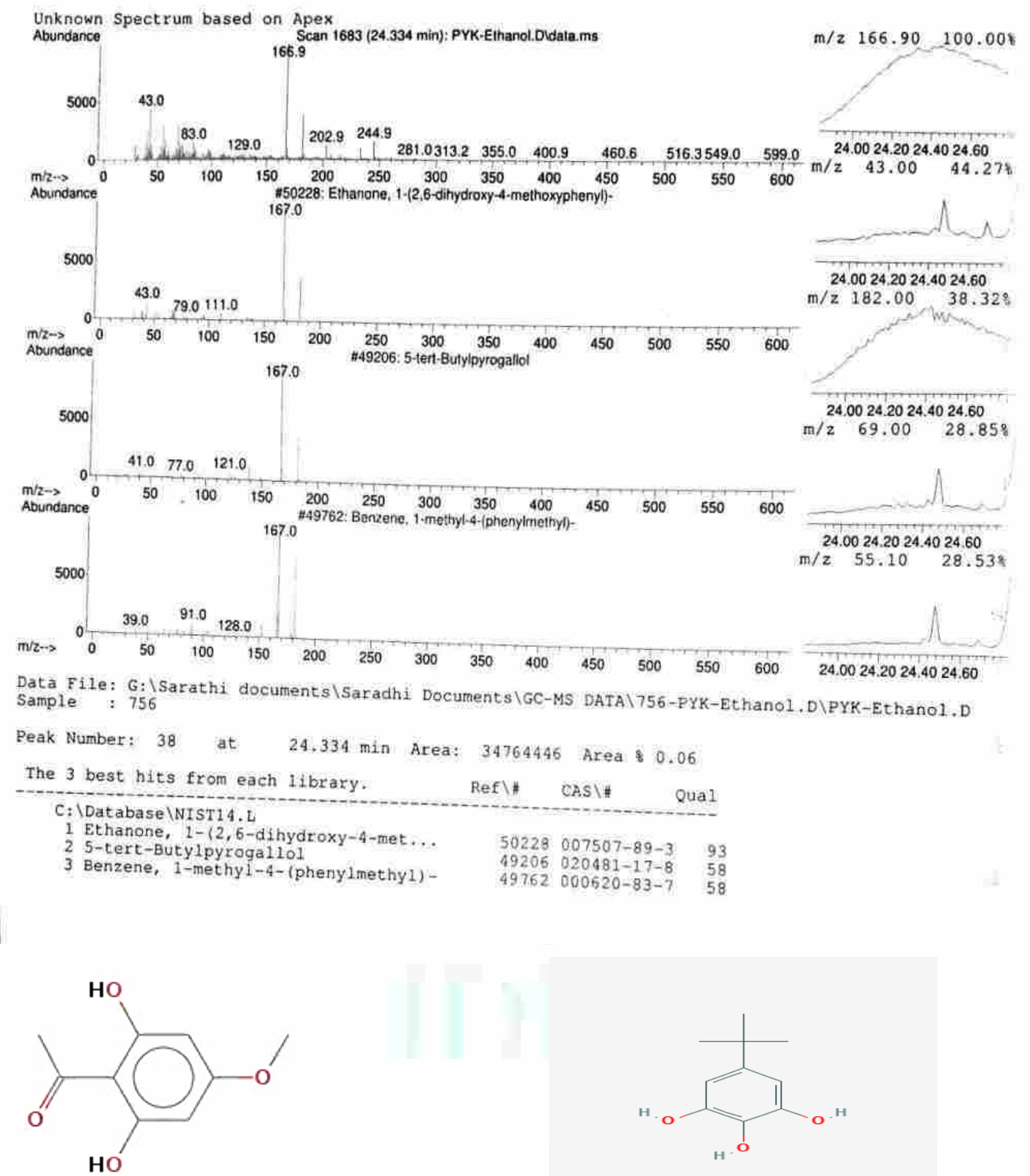

Ethenone, 1-(2, 6-dihydroxy-4-methoxyphenyl)-5-Tert-Butylpyrogallol 
Table 1. Phytocomponents found in pykure capsule by gc-ms analysis.

\begin{tabular}{|c|c|c|c|}
\hline S.NO. & COMPOUND NAME & MOLECULAR FORMULA & MOLECULAR WEIGHT \\
\hline 1 & 3,3-Diethoxy-1-propanol & $\mathrm{C}_{7} \mathrm{H}_{16} \mathrm{O}_{3}$ & $48.20 \mathrm{gm} / \mathrm{mol}$ \\
\hline 2 & 2-Pentanone, 4-hydroxy & $\mathrm{C}_{5} \mathrm{H}_{10} \mathrm{O}_{2}$ & $102.132 \mathrm{gm} / \mathrm{mol}$ \\
\hline 3 & Acetaldehyde, methoxy- & $\mathrm{C}_{3} \mathrm{H}_{6} \mathrm{O}_{2}$ & $74.01 \mathrm{gm} / \mathrm{mol}$ \\
\hline 4 & Carbonic acid, ethyl-, methyl ester & $\begin{array}{l}\mathrm{CH}_{2} \mathrm{O}_{3} \\
\mathrm{C}_{8} \mathrm{H}_{15} \mathrm{NO}_{4}\end{array}$ & $\begin{array}{l}62.024 \\
30189.211\end{array}$ \\
\hline 5 & Diethyl carbonate & $\mathrm{C}_{5} \mathrm{H}_{10} \mathrm{O}_{3}$ & $118.132 \mathrm{gm} / \mathrm{mol}$ \\
\hline 6 & Bicyclo[4.2.0]octa-1,3,5-triene & $\mathrm{C}_{8} \mathrm{H}_{8}$ & $104.152 \mathrm{gm} / \mathrm{mol}$ \\
\hline 7 & Benzaldehyde & $\mathrm{C}_{7} \mathrm{H}_{6} \mathrm{O}$ & $106.12 \mathrm{gm} / \mathrm{mol}$ \\
\hline 8 & Ethane dioic acid, diethyl ester & $\mathrm{C}_{6} \mathrm{H}_{10} \mathrm{O}_{4}$ & $146.14 \mathrm{gm} / \mathrm{mol}$ \\
\hline 9 & Propane dioic acid, oxo-, diethyl ester & $\mathrm{C}_{5} \mathrm{H}_{8} \mathrm{O}_{3}$ & $116,116 \mathrm{gm} / \mathrm{mol}$ \\
\hline 10 & Pentane-1,2,3,4,5-pentaol & $\mathrm{C}_{5} \mathrm{H}_{12} \mathrm{O}_{5}$ & $152.145 \mathrm{gm} / \mathrm{mol}$ \\
\hline 11 & D-Arabinitol & $\mathrm{C}_{5} \mathrm{H}_{12} \mathrm{O}_{5}$ & $152.246 \mathrm{gm} / \mathrm{mol}$ \\
\hline 12 & 3,3-Diethoxy-1-propanol & $(\mathrm{CH})_{3} \mathrm{CCH}_{2} \mathrm{OH}$ & $88.15 \mathrm{gm} / \mathrm{mol}$ \\
\hline 13 & Propane, 1,1,3,3-tetraethoxy & $\mathrm{C}_{7} \mathrm{H}_{8} \mathrm{O}_{8}$ & $220.133 \mathrm{gm} / \mathrm{mol}$ \\
\hline 14 & 1,2,3-Butanetriol & $\mathrm{C}_{4} \mathrm{H}_{10} \mathrm{O}_{3}$ & $106.121 \mathrm{gm} / \mathrm{mol}$ \\
\hline 15 & 5-Hydroxymethylfurfural & $\mathrm{C}_{6} \mathrm{H}_{6} \mathrm{O}_{3}$ & $126.11 \mathrm{gm} / \mathrm{mol}$ \\
\hline 16 & 2-Heptenal, 2-methyl- & $\mathrm{C}_{2} \mathrm{H}_{4} \mathrm{O}_{2}$ & $60.252 \mathrm{gm} / \mathrm{mol}$ \\
\hline 17 & 2-Propenoic acid, 3-phenyl ethyl ester & $\mathrm{C}_{11} \mathrm{H}_{12} \mathrm{O}_{2}$ & $176.125 \mathrm{gm} / \mathrm{mol}$ \\
\hline 18 & 4-Methylphenoxyacetonitrile & $\mathrm{C}_{9} \mathrm{H}_{9} \mathrm{NO}$ & $147.177 \mathrm{gm} / \mathrm{mol}$ \\
\hline 19 & Tricosane & $\mathrm{C}_{22} \mathrm{H}_{48}$ & $324.637 \mathrm{gm} / \mathrm{mol}$ \\
\hline 20 & Tetradecane, 2-methyl- & $\mathrm{C}_{15} \mathrm{H}_{32}$ & $212.421 \mathrm{gm} / \mathrm{mol}$ \\
\hline 21 & Ethanone, 2-hydroxy-1,2-diphenyl-, oxime & $\mathrm{C}_{14} \mathrm{H}_{13} \mathrm{NO}_{2}$ & $227.269 \mathrm{gm} / \mathrm{mol}$ \\
\hline 22 & 2-Bromo dodecane & $\mathrm{C}_{12} \mathrm{H}_{25} \mathrm{Br}$ & $249.236 \mathrm{gm} / \mathrm{mol}$ \\
\hline 23 & Dodecane, 2,6,11-trimethyl- & $\mathrm{C}_{15} \mathrm{H}_{32}$ & $212.421 \mathrm{gm} / \mathrm{mol}$ \\
\hline 24 & Hexadecane, 2,6,10,14-tetramethyl- & $\mathrm{C}_{20} \mathrm{H}_{42}$ & $282.556 \mathrm{gm} / \mathrm{mol}$ \\
\hline 25 & Acridine & $\mathrm{C}_{13} \mathrm{H}_{9} \mathrm{~N}$ & $79.13 \mathrm{gm} / \mathrm{mol}$ \\
\hline 26 & (3-Methoxy-2-nitrophenyl)acetic acid, methyl ester & $\mathrm{C}_{9} \mathrm{H}_{9} \mathrm{~N} \mathrm{O}_{5}$ & $211.173 \mathrm{gm} / \mathrm{mol}$ \\
\hline 27 & Pentatriacontane & $\mathrm{C}_{35} \mathrm{H}_{72}$ & $492.96 \mathrm{gm} / \mathrm{mol}$ \\
\hline 28 & 3-phenylprop-2-enoic anhydride & $\mathrm{C}_{18} \mathrm{H}_{14} \mathrm{O}_{3}$ & $278.307 \mathrm{gm} / \mathrm{mol}$ \\
\hline 29 & trans-1-Cinnamoylimidazole & $\mathrm{C}_{9} \mathrm{H}_{8} \mathrm{~N}_{2}$ & $144.177 \mathrm{gm} / \mathrm{mol}$ \\
\hline 30 & Undecane, 2-methyl- & $\mathrm{C}_{18} \mathrm{H}_{30}$ & $246.438 \mathrm{gm} / \mathrm{mol}$ \\
\hline 31 & Isopropyl octadecyl ether & $\mathrm{C}_{9} \mathrm{H}_{22} \mathrm{O}_{2}$ & $162.273 \mathrm{gm} / \mathrm{mol}$ \\
\hline 32 & Docosane, 7-hexyl- & $\mathrm{C}_{28} \mathrm{H}_{58}$ & $394.772 \mathrm{gm} / \mathrm{mol}$ \\
\hline 33 & Eicosane, 10-methyl & $\mathrm{C}_{21} \mathrm{H}_{44}$ & $296.583 \mathrm{gm} / \mathrm{mol}$ \\
\hline 34 & Hexadecenoic acid, methyl ester & $\mathrm{C}_{17} \mathrm{H}_{34} \mathrm{O}_{2}$ & $270.45 \mathrm{gm} / \mathrm{mol}$ \\
\hline 35 & 8-Methoxy-[1,2,4]triazolo[4,3-a]pyridine-3-thiol & $\mathrm{C}_{6} \mathrm{H}_{5} \mathrm{~N}_{3}$ & $119.127 \mathrm{gm} / \mathrm{mol}$ \\
\hline 36 & 2,2'-Dimethylbiphenyl & $\mathrm{C}_{14} \mathrm{H}_{14}$ & $182.144 \mathrm{gm} / \mathrm{mol}$ \\
\hline 37 & 4-Ethylbiphenyl & $\mathrm{C}_{12} \mathrm{H}_{14}$ & $182.266 \mathrm{gm} / \mathrm{mol}$ \\
\hline 38 & Hydroquinone, TMS derivative & $\mathrm{C}_{6} \mathrm{H}_{6} \mathrm{O}_{2}$ & $110.11 \mathrm{gm} / \mathrm{mol}$ \\
\hline 39 & Benzene, 1-methyl-4-(phenylmethyl)- & $\mathrm{C}_{8} \mathrm{H}_{10} \mathrm{~S}$ & $138.228 \mathrm{gm} / \mathrm{mol}$ \\
\hline 40 & 5-tert-Butylpyrogallol & $\mathrm{C}_{10} \mathrm{H}_{14} \mathrm{O}_{3}$ & $182.219 \mathrm{gm} / \mathrm{mol}$ \\
\hline 41 & Ethanone, 1-(2,6-dihydroxy-4-methoxyphenyl & $\mathrm{C}_{9} \mathrm{H}_{10} \mathrm{O}_{3}$ & $185.175 \mathrm{gm} / \mathrm{mol}$ \\
\hline 42 & 2-Nonyl-1-ol, diethyl acetal & $\mathrm{C}_{11} \mathrm{H}_{24} \mathrm{O}_{2}$ & $188.311 \mathrm{gm} / \mathrm{mol}$ \\
\hline 43 & 9-Octadecenoic acid (Z)-, methyl ester & $\mathrm{C}_{13} \mathrm{H}_{24} \mathrm{O} 2$ & $212.333 \mathrm{gm} / \mathrm{mol}$ \\
\hline 44 & 11-Octadecenoic acid, methyl ester & $\mathrm{C}_{19} \mathrm{H}_{36} \mathrm{O}_{2}$ & $296.495 \mathrm{gm} / \mathrm{mol}$ \\
\hline 45 & Octadecanoic acid, ethyl ester & $\mathrm{C}_{20} \mathrm{H}_{40} \mathrm{O}_{2}$ & $282.48 \mathrm{gm} / \mathrm{mol}$ \\
\hline 46 & 1,1'-Biphenyl, 2,2'-diphenoxy- & $\mathrm{C}_{12} \mathrm{H}_{12} \mathrm{~N}_{2}$ & $184.242 \mathrm{gm} / \mathrm{mol}$ \\
\hline 47 & 17,21-Dimethylheptatriacontane & $\mathrm{C}_{39} \mathrm{H}_{80}$ & $549.069 \mathrm{gm} / \mathrm{mol}$ \\
\hline 48 & trans-Geranylgeraniol & $\mathrm{C}_{20} \mathrm{H}_{34} \mathrm{O}$ & $290.491 \mathrm{gm} / \mathrm{mol}$ \\
\hline 49 & S-(-)-1,2,4-Butanetriol, 2-acetate & $\mathrm{C}_{4} \mathrm{H}_{10} \mathrm{O}_{3}$ & $106.121 \mathrm{gm} / \mathrm{mol}$ \\
\hline 49 & Styrene & $\mathrm{C}_{8} \mathrm{H}_{8}$ & $104.152 \mathrm{gm} / \mathrm{mol}$ \\
\hline 50 & 1,3-Butanediol, (S)- & $\mathrm{C}_{4} \mathrm{H}_{10} \mathrm{O}_{2}$ & $90.122 \mathrm{gm} / \mathrm{mol}$ \\
\hline 51 & S-(-)-1-Phenyl propanol & $\mathrm{C}_{9} \mathrm{H}_{12} \mathrm{O}$ & $136.194 \mathrm{gm} / \mathrm{mol}$ \\
\hline 52 & 1,5,6,7-Tetrahydro-4-indolone & $\mathrm{C}_{8} \mathrm{H}_{9} \mathrm{NO}$ & $135.168 \mathrm{gm} / \mathrm{mol}$ \\
\hline 53 & Benzaldehyde diethylacetal & $\mathrm{C}_{11} \mathrm{H}_{16} \mathrm{O}_{2}$ & $180.247 \mathrm{gm} / \mathrm{mol}$ \\
\hline 54 & Trans-Cinnamic acid & $\mathrm{C}{ }_{9} \mathrm{H}_{8} \mathrm{O}_{2}$ & $148.158 \mathrm{gm} / \mathrm{mol}$ \\
\hline 55 & Tridecane,7-hexyl- & $\mathrm{C}_{19} \mathrm{H}_{40}$ & $268.529 \mathrm{gm} / \mathrm{mol}$ \\
\hline 56 & n-Hexadecanoic acid & $\mathrm{C}_{16} \mathrm{H}_{32} \mathrm{O}_{2}$ & $256.4 \mathrm{gm} / \mathrm{mol}$ \\
\hline 57 & Resorcinol, TMS derivative & $\mathrm{C}_{6} \mathrm{H}_{6} \mathrm{O}_{2}$ & $110.1 \mathrm{gm} / \mathrm{mol}$ \\
\hline 58 & 8-(2-Nitrophenoxy)octan-1-ol & $\mathrm{C}_{14} \mathrm{H}_{21} \mathrm{O}_{4}$ & $267.325 \mathrm{gm} / \mathrm{mol}$ \\
\hline 59 & Eicosane,2,6,10,14,18-pentamethyl & $\mathrm{C}_{25} \mathrm{H}_{52}$ & $352.691 \mathrm{gm} / \mathrm{mol}$ \\
\hline 60 & Oleic acid & $\mathrm{C}_{18} \mathrm{H}_{34} \mathrm{O}_{2}$ & $282.47 \mathrm{gm} / \mathrm{mol}$ \\
\hline 61 & 1-Bromo-11-iodoundecane & $\mathrm{C}_{11} \mathrm{H}_{23} \mathrm{Br}$ & $235.209 \mathrm{gm} / \mathrm{mol}$ \\
\hline 62 & tert- Hexadecanethiol & $\mathrm{C}_{48} \mathrm{H}_{99} \mathrm{AuS}_{3}$ & $969.467 \mathrm{gm} / \mathrm{mol}$ \\
\hline
\end{tabular}




\begin{tabular}{|l|l|l|l|}
\hline 63 & Supraene & $\mathrm{C}_{19} \mathrm{H}_{10} \mathrm{Br}_{4} \mathrm{O}_{5} \mathrm{~S}$ & $331.35 \mathrm{gm} / \mathrm{mol}$ \\
\hline 64 & Isopropyl octacosyl ether & $\mathrm{C}_{4} \mathrm{H}_{10} \mathrm{O}$ & $74.123 \mathrm{gm} / \mathrm{mol}$ \\
\hline 65 & 2-Methyltriacontane & $\mathrm{C}_{3164} \mathrm{H}$ & $436.853 \mathrm{gm} / \mathrm{mol}$ \\
\hline 66 & 2-Methylpentacosane & $\mathrm{C}_{16} \mathrm{H}_{34}$ & $226.448 \mathrm{gm} / \mathrm{mol}$ \\
\hline 67 & Squalene & $\mathrm{C}_{30} \mathrm{H}_{50}$ & $410.73 \mathrm{gm} / \mathrm{mol}$ \\
\hline 68 & Hexadecane, 7,9-dimethyl- & $\mathrm{C}_{18} \mathrm{H}_{38}$ & $254.502 \mathrm{gm} / \mathrm{mol}$ \\
\hline 69 & Triacontane, 11,20-didecyl & $\mathrm{C}_{50} \mathrm{H}_{102}$ & $703.366 \mathrm{gm} / \mathrm{mol}$ \\
\hline 70 & Nonadecane & $\mathrm{C}_{19} \mathrm{H}_{40}$ & $308.773 \mathrm{gm} / \mathrm{mol}$ \\
\hline 71 & Disulfide, di-tert-dodecyl & $\mathrm{C}_{24} \mathrm{H}_{30} \mathrm{~S}_{2}$ & $402.787 \mathrm{gm} / \mathrm{mol}$ \\
\hline 72 & Tricosane & $\mathrm{C}_{23} \mathrm{H}_{48}$ & $324.637 \mathrm{gm} / \mathrm{mol}$ \\
\hline 73 & Eicosane & $\mathrm{C}_{20} \mathrm{H}_{42} \mathrm{O}$ & $282.529 \mathrm{gm} / \mathrm{mol}$ \\
\hline 74 & Hentricontane & $\mathrm{C}_{31} \mathrm{H}_{64}$ & $436.85 \mathrm{gm} / \mathrm{mol}$ \\
\hline
\end{tabular}

\section{CONCLUSION}

Ayurvedic herbal formulations are widely used in allopathic era for their effectiveness and reduced risk of side effects. Pykure is a poly herbal capsule containing Mesua ferrea, Terminalia chebularates, Cuminium cyminium, Azadiracta indica, Saraca indica, Abutilon indicum, Picrorhiza kurroa and Red ochre. Label claims different dosages for varied pharmacological activity in Bleeding hemorrhoids, fissures and rectal swelling, prolapsed of rectum, fistula. For quest of compounds present in poly herbal formulation. Spectroscopic studies mainly Gas chromatography and Mass spectroscopy was carried out and 74 known compounds were reported and matched with NIST library of IICT.

\section{ACKNOWLEDGEMENT:}

I would like to thank Mr.Vijaya Sarathi, Sr.Scientist IICT, Tarnaka, Hyderabad, Telangana for providing the facility and support used to carry out the work.

\section{REFERENCES:}

1. http://www.holistic-online.com/Herbal-med/hol_herbintro.htm

2. https://www.pharmatutor.org/articles/review-qualitycontrol-herbal-drugs

3. https://www.mydr.com.au/complementary-medicine/herbalmedicine

4. https://www.indiamart.com/proddetail/pykure-capsules-forpiles-2172090130.html http://www.herbalbodyindia.com/indications.html

6. Seema Firdouse, jyoti gupta, Parwez Alam, GC-MS Analysis of Amorphophallus Campanulatus Decne Tuber. American journal of pharmtec research Vol. 6, issue 3, 2016, 791-797. 